$\mathfrak{D} \mathfrak{r}$

\title{
Deutide Geiftesarifotrationus.
}

\author{
Ein $\mathfrak{B}$ eitrag
}

ว $\mathfrak{x}$

Egaralteriftie bes geitigen politiffen

Geifteg

$\mathfrak{i} \mathfrak{D} \in \mathfrak{u} \mathfrak{t} \mathfrak{f} d \mathfrak{l} \mathfrak{a} \mathfrak{n} \mathfrak{b}_{\text {. }}$

$\mathfrak{B} \bigcirc \mathfrak{n}$

D r. $\Theta_{.} \dot{\mathfrak{H}} \boldsymbol{f} \mathfrak{d}$ e r. .

Amicus Plato, amicus Aristoteles,

sed magis amica veritas.

\section{Let $\mathfrak{i} \mathfrak{i g}, \quad$ I 819}

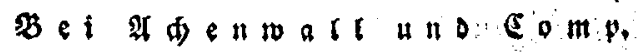




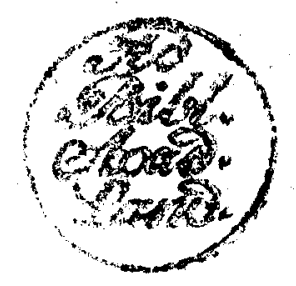

भI

tion ausbrad, gab man die Philorophen, Eno c) Hlopásiften, Detonomiften und Philantbros pen nlอ bie Hrbeber, ober zum wenigften alకె Die veranlaffende lurfadse berfelben an., Nages

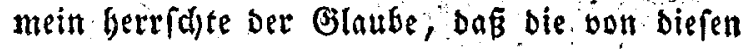
Beiftern verbreiteten Soeen und Infiditen eine Stimmung in allen Etănden ber Bolfb̉mafie verórcitet, wosurd bie Shritte berjentigent

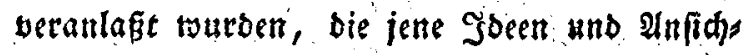
tett zu verwirflidien făbig waren.

Im Granise fpradent die Denfenden- Idpfe nur beftimmt uno Elar bie Soen uno Infid)ten

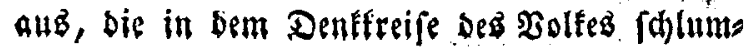


merten. W3ie wåre es fonft móglid seween,

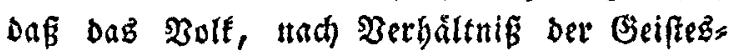
entwicfelung feiner verfdiebenen Stånbe, mefr ober weniger fie aufgefapt, begünftigt uno fid) won ifnen bătte leiten lafjen. 2alle Propheten, Sefejgeber, Staatengetúner haben Daburd) nur cinen (Einfut auf bie Jienge erbals tent und fie nad) ibren abfidten leiten fơnnen, weil fie bis Geiftegentroictelung berfelben unb bie in jebem 2olfe (c)lummernden Joeen an= fpradien. St)werlich Dirften die univerfellen Nôfe einer taujendjăbrigen Borgeit, eit $\mathfrak{R}_{0}$ fez, Zoroafer, Mabomet, eil Solon, Zaleutub, Romulus unb Rarl ber Gros Be fo tief in Die Gemútblid) feit eines ber zeitigen Alera eingreifen, aloz vormalz, unb eben fo fteril whirbe auth ber cintitus gewefett

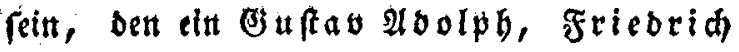
Det Grofe, Sofepb ber Zweite, Frant= Iin unb felbft Rapoleon anf ein alterthüms liches $\mathfrak{B}$ olf gehabt baben buirfte.

Die benfenben నiōpfe eineş Bolfez̧, mơgen

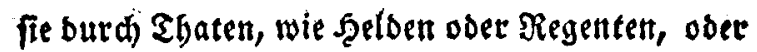
Durd) Sdriften, als Plilofopben uno Dichter wirfett, ftellett immer bas Refultat ber anlage auf, bie jene nidjt faffien, fonbern blos faneller entwidfelt.

Man ift baber immer in einem tiefen Sres

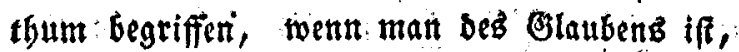
Dá ein ganjez $\mathfrak{B}$ olf von einem eminenten Sopf outd Sbat ober Sarift irre seleitet wers: ben fonne. Eg ift bie Stimmung, bie Grands. notive ber Soltsgenútblichteit felbft, bie bet eminenten Ropf unterftuge unb trăftigt, Eit eminenter Ropf bat unfeblbat einen boin ber Slfmad)t, ober went ibr es হaturnennen wollt; vorberbeftimmten Seitfaben für leine Wirffam: Eeit. Mag er fein Bolk zum Gutent ober zum Sd)ledte:-binleitent. Sein Streben trågt nie allein bie Sauld. Eitt vorberbeftimmtes uts

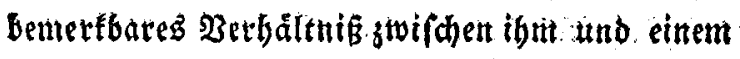
Bolfe tritt in feiner Betriebjamfeit nut berbor.

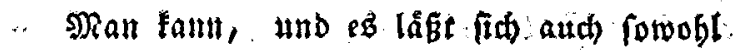
aแถ ben Innalen ber Yor\}eit, als ben-Bor: găngett unferet 3eit erbártent, (ể leidjt ets. meffen: ob bas Treiben eittes attsgeseidneten

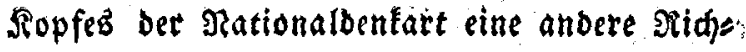
tung zu verleiben, fo funbirt unb geeignet iff, piner Nation ober Menfdenmenge allgemeine

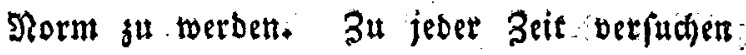
eminente Geifter aus̉gejeiantet ju wirfen, al lein niche immet wird ibr beftrebent von ber Bolfzftimutung fo belobnt, baß fie einente: folgreident Eimflus erwartent burfen.; \$ar Shaffen unb Sreibet wird Dann ein Gegens: Ranb ber פeadfung ober ber Disfuffion, und 


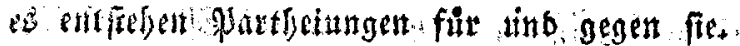
Der Siamp ber \$artbeien gegen einander ento. fojcibet für ober gegen ben Sleformator, atts bam iff Red)t bes Etárferen, welches ben Ausfálag: giebt. Dasi গiefultat, weldhes baraus. hervors. gebt, it if bunn nidit imute ber wabre anbs: fprud ber Nation, ober bab, wab die Entwies:

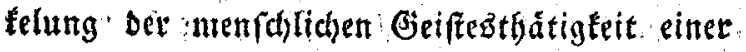

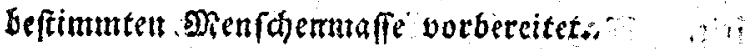

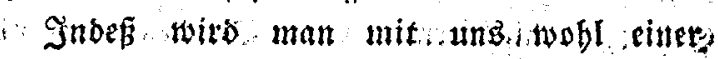

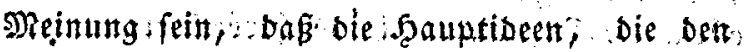
Segebenbeiten ber. frat jó fifchent Sevolution;

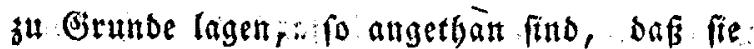
gleidffant itt bem Gemulth ber frangofifden Nation fhluntmerten, uno als fie ifrevon ifren:

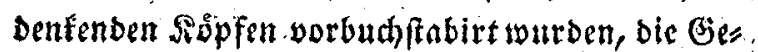
muither baburd twievoneinem eleftriß aufgeregt wurden. Die $\$$ artheiurgen, bie fie it ifs: remsereife aufregten, entwudd fen sicht dem $\subseteq$ to ff Det Joent, fondern bildeten fid exff, als man

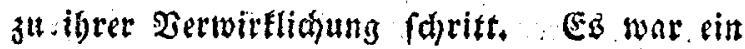
STampf um Die. ซorm.

Mit Dem Entféten und bem untergange det mandjerlei \$artbeien im revolutionniren Fra a

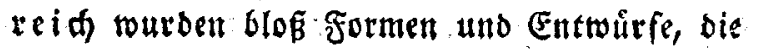
grofen Joeet Der. Staatzroiffenfdaft,

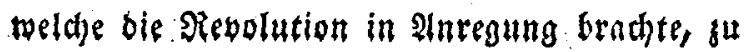

realifien, Ja Geabe gefradit, Der faatbiste

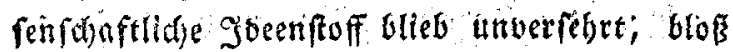
bie Sorm, in welder er erbalten werben poin, nóa ber Jegenftand ber Gebbe. . . .

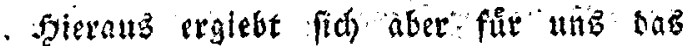
g:of́e Sefultat, Dafi dic Soent, weldje ber frang ofif

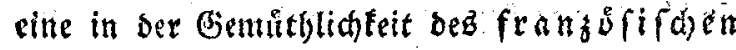
Solteg fundirte Geiffeşbaltung bildete, bie

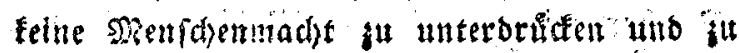

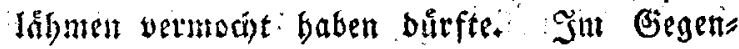

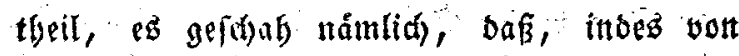
affen Seiten ber bie Siegierunget eas verfudten,

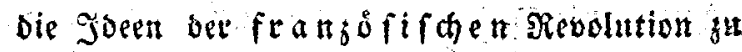
befămpfen unb gu unterbrîd

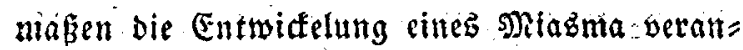
Ingten; bas ben Brund ju einer Beteftesserwegung in Europa legte; bie in ben feften Inftitutio: then beffilben eine Erjoutterung vorbereitete.

Soetn man aber ben Einfus, den bie frangofifhe Mevolution auf bie verfaleber nen $\mathfrak{B}$ dffer und Staaten batte, fararf in's Aige fagt, fo wiro man bod bentertest, dafsgenau genommen, ber Einfuñ : ber revolutio: nairen Soeen nidt fo tief in oie Gemuthlids. feit der' Rationen eingriff, als auf bie ber frăa= źfifden Nation. Sei sinem jeben europáis

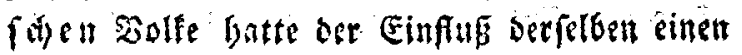




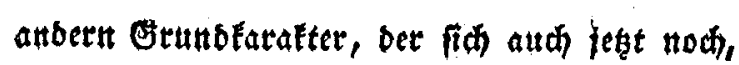
nachoem bie Rebolution att ibrer Duelle ein Biel erreicht ju baben fheint, bei allen fo jiem, (ich) aubbrũdf. Stalien war immer gleid) fam ber Stlave der SRevolutionbiben gewefen, bie es wieber fabren liek, als ez won peinen settent

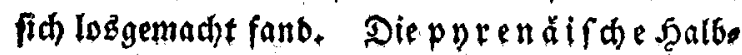
infel (Spanien und Portugall) batte mit ibnen einen exteffaften Sampf ausgebalten. Sif bat, twie ein beberster Glabiator, fich wott bemfelben gleiafam wieder erbolt, unb nimmt nut ibe vorige Stellung beinake wieber ein. Englanb toebte, wie ein fråftiger \&ơwe, mit

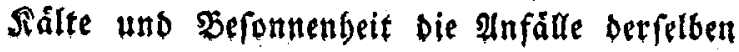
fid ab. Şolla nb bat, wie sin geubter Raufs mant, angenommett und gezaflt. Der falwei= jerifare Geift blieb feft, wie feitte unubers fteiglidse Hrpen, Şm Morben Europas, in Dánnemare, Squeben und Rukland fand baß̧ regolutionaire Pblegma wenig Theile nahme, Ein anderes war es aber mit Det t $[$ d lanb, diefer feimath univerfeller Geiftebful fur. Deutfhland foreint gleidylam oer Brennpunt fut feitt, itt weldem fich alles Streben geiftiger Entwickelung ber europåi= faen Nationen fammeln, unb wo bin uno wieber bas gơttlide Jetuet ber Soeen jur నlars beit und ieinbeit gefteigert werben foll, $D a=$

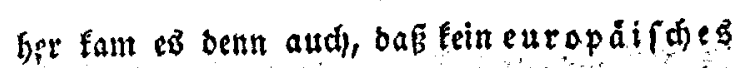

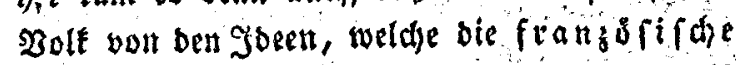
revolution bafirte, fo tief und umfaffend, als bas geiftige Raturell ber Deutfhen, ergriffen warb.

Das geiftige Saturell fage iá. Denit Gierin beftebt eben bas Gigcutbümliage ber Sheilnabme Deutfdland an ser frango: fifhen Revolution, baf bie goen, die ifs zu Grunbe liegen, uno welche ben Stoff bez: jenigen, was wir Staatbifoiffenfaaft jetht nennen, bilben, bloģ von ben benfenden Soffen aufgeftell, fultivirt und in bem Grabe im literaris

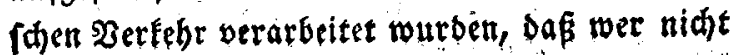
ein naber Beobachter bes \&ebens und ₹reibenz it Deutfd! a no war, fu wăbnen in গerfuchung geratben fonnte, ber Fevolutions getf babe bereits in allen Gauen bafelgft oen Buftant ber Din ge in Feuer uno Flammen verfegt. So lebbaft, vielfeitig, partheigångerartig verhanbelte man über alle, in Der Revolutiongett in Frant" reid) jur Sprache gefommene Soeet, und Erfarinungen und Begebenbeiten, bie fie er: bárten ober berwirflid)en follten, in mandiers lei Werten, Sd)riften, Srodựren, Pamphletż, Zeit $=$ und Tagebláttern.

WBer Augenjeuge doer gar Theilnehmer beş literarifhen 2renebrs mit politifhen Soben, 


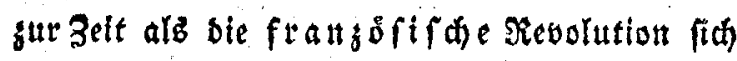
entfaltete, war, ber wirb wobl eiturălument mulfen, baf aufer Frantecta feloft, teitie

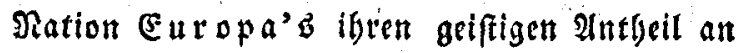
ber lumwälung franfreid s burd) eitte fols the Angabl Drudfidififten manifeftirte, als Deut (d)lano.

Der gelefrte Stanb war es alfo, in

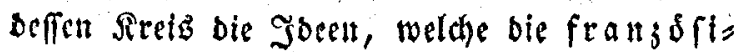
fdre Revolution anfegte, cinen Stofi abgas ben, ser cinen grofen Thell feinet beiftesthas tigfrit in Slufpruch nafm.

San bente fich aber aud ben lumfang beb

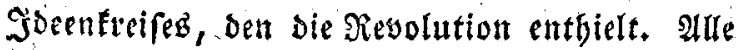
yofitiven Sinftitute bes Statü, Religionswer faffung, bargebracite Siechite uno von IIterb ber gefoiligte Sitten rurben in ber Retorte ber politifach @dheibefinfler, Durch bie, Jjoen, oon walden fie begeiftert waren, analufit uno

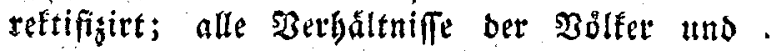
Stäten warben nad ben Beredinungen uno Foruteln idealifaler Sozmopoliten und \$gilans figropen bypeftafirt uno normirt, unb alfe biefe Dperationen unb Experimente muftett vorghislid) Den fpefulativen Soppen in Deut $j$ d) Iano einst unerfhopplidjen Stoff jur Dears beitung barbieten.

Wab biefe literavifitge Detriebfamfeit nod mebr unterfinte, wat bie fpefulative sida - tung, welde bie fritifde philofoplie in ben achtziger Jabren bes vorigen Setulumb bett Deuthen bentenden Topfen gas. Jn Dan

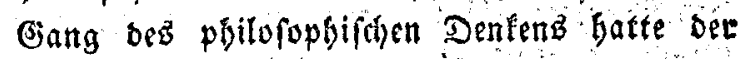
Ed)wpfer bes Rutifis mus etne Revolution veranlaft, bie fich aber alle sroetge dea swip fenz in Deut (d) la no verbreitet Gatte. Soen; Iinfichtent, Methoden unb Bortrag in Sireife

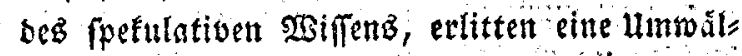
zung, yon toldfer berjonige nue eine lebbafte und vollentete Borftellutg baben tann, ber Siefe Seriode der belitfden Riteratur mit sers lekte. Er allein fann ex ermeflen, wie the fie nuf bie Denfendet Sopfe Deutfdilanba eins griff und rsie cmpfänglich fie felbige fitr bie foes

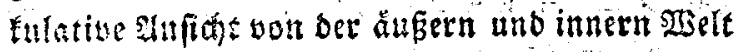
glcichiom biloste.

Dev Sutigismus hatte bas Eigenthüm lide zugleid, bafi er bie Spilofopbie su einer cigentlid)en sisiffenfant wieber erbob." Er rettete fie aus bem Strome ber $\$$ lopularitat, in welche fie eine $\mathfrak{I m}_{\mathfrak{a}}$ abl fladher, empfinderits ser uns von der Sinnlidfelt beraufder $\Re$ opfe bineingefruselt, bie fid feftr weifs und tiefs

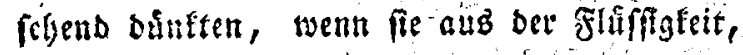
in weldie fie allfgelopt worben, getwiff pattielle

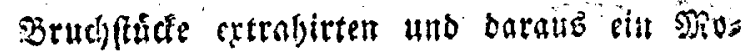


fail afluáglig biloeten, bas fie stoalitions: poilofopbie namten.

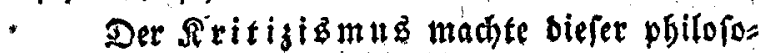
pbifaren Reseptirfunft ben Garaus, unb erbob bas fpefulative $\mathfrak{w i f f e n t}$ auf eine feitter wurbige Stufe. Freilid ging es bei Diefem Jerfabren nidat gat frieblid ab. Die Popularpbilo= fopben exboben laut gegen eine Metbode, bie ibre gefdumulate, geregelte und aefthetifa) spa= niex zertrümmerte, ibre Stimmett, Sie ers

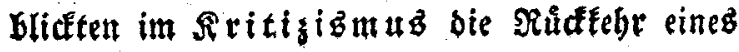
fđolaftifden 3ettalterbె, burd) weld)ę bie Pbis Iofophie, bie fie bon bem Simmel in ben Ges fellfałaftżfreis herabgezaubert ju baben fich tâbmten, it bie bóbern গegionen abermalb werítheudt werben mún. Sie wábnten fdon bas Beitaltet bet Dialeftifer unb $\mathfrak{T}$ heofos

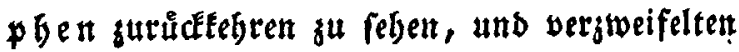
an bein Forţđritt ber beutfden Geiftę; bilbung.

Denn wabr ift $\mathrm{eB}$, wer bie Geiftesperodufte bamaliger 3 eit reât ins 2luge faßst, oce wirb co iften abmerten, baf bie tritifa) Pbilofo: phic ben måd)tigften Finfuß auf Soeengang uno Bortrag ůbte, Frembartige Fusbrũcte, für weld)e bei ber Iuffaffung neuer Joeen unb beren Rombination fid nidit fofort vaterlăt: bifbe Senentungen vorfanben, Bebrängtbeit unb Srodfenbeit im Portrag, cine Folge yout ber Siefe und Abftrufitåt ber Gebanfen, mit welchen diefe Pbilofophie ben Denfer bereidjerte, Den Beift and bie Aufnerffanfeit ergreifende Syntberen und 2 (naly)en ber Begriffe, worin Die Eigentfümlithteit des Rritigismas fid vorğglich aublprach, alle biefe Eigenbeiten mubten es wobl veranlafien, Dab die literaris (d)et Probufte, welde allb Dem Sreife ber allgemein verebrten tritifden Shule bervor: gingen, die Menge opn Den Suellen ber Weib: beit, weldhe die popularphilofophen un fie bee verfammelt gatten, furudifheud)ten.

Indef mú man bod, wobl anerfenten, went ber in Dept (d)land allgemein aufges regte ઉeift ber Epefulation an 3 reite werloren, er bod getwiffermasen an Tiefe unverbált nifmásig dagegen gewann, uno man fonnte

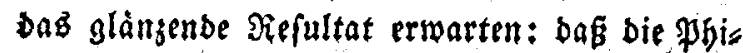
lofophie ober die ipefalative Infiat ber Joeen, welde das Gebiet Der Notbrondigteit unb Freibeit bilden, nunmebr feiner wabren $5 e=$ ftimmung gemå $\mathfrak{\beta}$, biejenigen. Beifter nut it ifre Regionen werbe cintreten feben, welde.

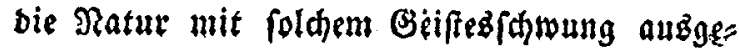
ftattet, um auf ber . Seibe ber Spefulation fich emporbalten zu fơnnen. Es war ju bofien,

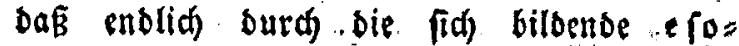


terifde Geifestaltung unter ben bentenbers

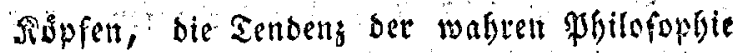
crfantu, feftgebalten; uts nid) in ben 230 ort: frhivall ber viel bin ttto ber rebenden $23 e r$

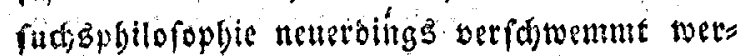
ben burite.

Nach meiner $\mathscr{A}$ tridit if mant baber gants irriger Meinung, wenn man glaubt, osc sie Eritifare Syblofopble unmittelbar cinen Sbrits nebmer in bem Jpeengang bes gebilbeten Sheils Dentidland theil, tach ber Natur unb Beftaltung biefar Shbilupoplie, mufte "man vielmegr erwarter, Daß fie Das wabre Pbiloropgiren auf ben Sireiz ber von ber sRatul mit Denffraft begabten, siberen Geforianfen wurbe, unb bab sie sigents ridne. Philofophis witber in ben Girenzen fid nut regen ullo bewegen sulfte, wo fie in bett Beiten ibrer bidffen Slüthe fid gefialtet, id meine: in bein unfang ber philofopbifden $\mathfrak{S e d r a ́ l e ́ . ~}$

Dies wåre aud unfiflbar ber gall gewes fen, wenn um eben bie Periobe, als fuir

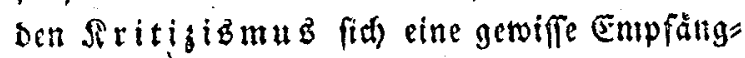
lichfeit in ben Denfenden $\Re$ offen $D e u t(d)=$ landz ausbilbcte, nidjt jugleid) bie Goeen, welche bie franzdiface Revelution, als Fundument cinet Staat 3 wiflenfalaft, in fojuelfen unb thatigen Hmlauf fegte, sie Mufs metrfamfeit beutfore Denfer auf firh gejo

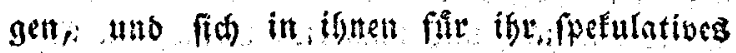
Patmel nidht ein reingaltiger Etoff Darges botan bätte.

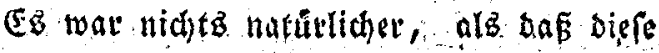
Naffe politifaer Joen auf den goengang ber

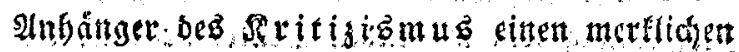

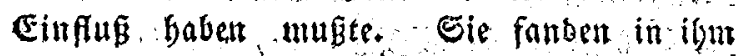
cinetr reidgaltigen Stoff, ben fie nach ben Srintpien ifrer Edjute ju bearbeiten begans nen uno an bem fie ibren Edjarffina ats wiven ourd, bent Oeift ber zeit gleidfam be ftârft wourden.

... Die Spefulation, welde auf Dem Cebiet ber theoretijtent Whilofopbie, ifren Spielraum sorgiglid) batte, fand num ein Sinterefie, int Bebirt ber praftifden Pbilofophie iffe Betrieb. fomfeit gu üben, und fo entroickelte fich it dent

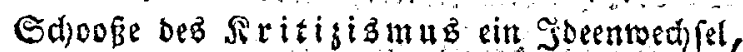

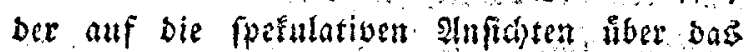

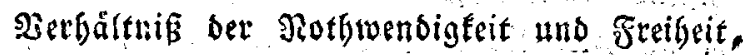
ber Natur unb Sittlidjeit cinen unbered)en: baren Finfug batte.

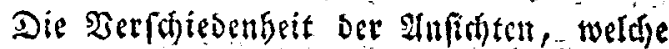
fid bilbete, veranlafite cinen gewiffen Ens

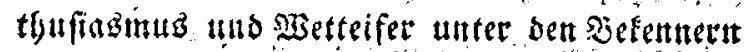
und Derfedtern Des feritioismub, fie ju 
vervollfomnenet uns fich nls bie Schöpfet. eines feften Funbamentz berfelben auffuftellen. So traten nad und nach sine Retibe vott Sy:. femidjo̊pfert auf. Ein Syftem tyard gleidfant yon bemt anbern betbrängt, ober feierte feinell Sriumph auf bett Srummetn feimeb $\mathfrak{B}$ orghts gers. Segion fonnte mant beinabe bie $3 a \mathfrak{s}$ bet felbfftåndig fein toollenbent Denfer thennen, bie

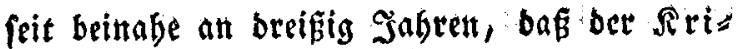
tigismus in Dentfalati ben Gant bes philopphifhen Geiftez leitete, auf ben $\mathbb{R}$ as

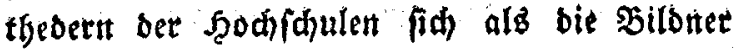

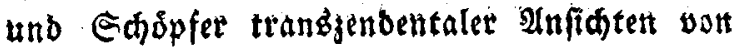
bem Betiet ber Natur unb Sittlidfeit auf:

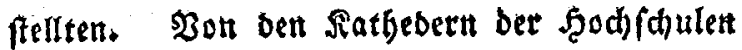
ging baher borgugstwetfe ber Entbufiastmus

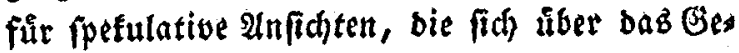
bitet ber æatur unb Sittlid feit erftedten, aus.

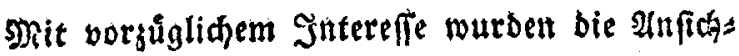
ten úber bie praftifdse Seite bes pefulativett soiffens fultivirt, ba bie Borgånge ber Re=

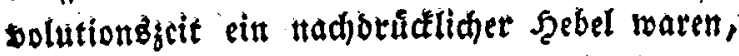
bas. Augenmert ber Denter auf bie ifr jut Grunde liegenden Soben fut leiten.

Şierburh warb abor in bem Gemuth bet betutiden Sugenb ein politifher Geif auf geregt, fứ bett fie immet ein groferes S̃ntes reffe gewann, als bie Ereigniffe, weldie bie
Revolution berbeifübrte, auf ben politifhen Juftano Deut Dendett Cinflü getwantent.

... Die Art uno 2 Baife indé, wie fich bet politfiche Geif in Deutfalans entwidelte, gab ifm einen gang eigenen Sarafter, ber ifit bon bemjenigen, wie er fich bisfer in Den pos litiphen Welthånbeln manifeftite, metflid) uns terfcheiden lå̉t. 2LB̈ Probuft ber Sđule bes bielt et inmet cine gemiffe theoretifde Jaal tung. Seine reglamfeit und Sorwegliafeit

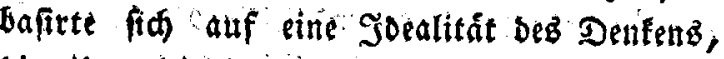
bie ifn gleidfan berbinberte, ins praftifde

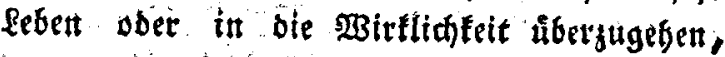

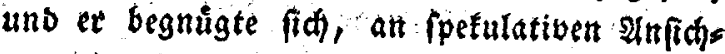

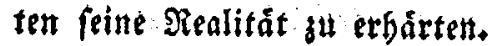

$\mathfrak{B e c}$ nut etwab mit ben Erfasinungent ber beutfónen philopopifdent Siteratur feit breigig Sabrett befretubet if, Der wirb bit Denertuth richtig finden, baß bie meifen Pros sutte berfelben benjenigen ift Dafein verbanten; welche bie Satheber ber Shod) (d)ulent inne batter,

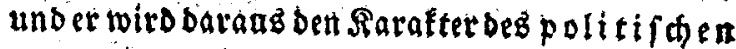
Geiftes ermeffet, von = weldsem bie Deutfde Jugend thuE befeclt toorben fein, und fernet att $b a z$ Refultat beraublommen, ba隹 fa barauz bie Ridjtung toiro beuten laffen, wels

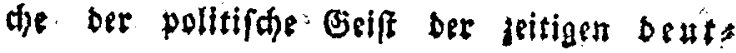


17.

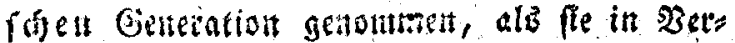
fuchung serteth, mit ocmplose ins praftifue febert cinjugecifer.

Ez: wirs tno muß aแb bent Gefagten it

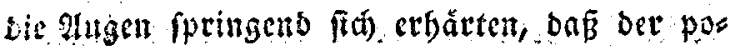
litifde Geift, Der in Deut falanofids ents wiofelte, nicht von ber Sation ober Der. Dent: alt bes Bolfes ansigitg, fondern originait in ber empfängliałen Gemúthỏat mefrerer, an ben

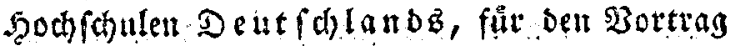
Der fpetulativen Shilofophie berufenet Mằ

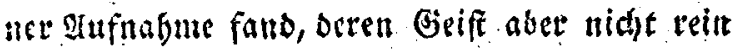
uns ubefongett, bem wirflider Reben befreun set twat, fondert der grdfitentheils mit ben

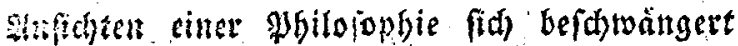
batte, weld)e, wie ber sititismab unb Die aus ifm beroorgegangenen Syfteme, in bem Gebiet Der . Freifeit, die \$rosleme ber tícactir fden Terff gu lofen fid berufen glaubte.

Dies gat Dexanlaffung, onfi in De

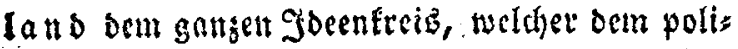
tifd)en Beift, ber bie Bölfar aub bent füblidjent Euxopa anbandite, fur $\mathfrak{D a f t s}$ biente, eine geniffermafet erfúgete Semperatur fu. Ebril warb. Man verwies alle politifale Joeen auf eitse gefteigerte Potens, uno fuć)te ifre Realitát allo eben bem Stanbpunft, meland bie fpefus

Iative
Iative Scite bev smotal und Religion oarbet, flt bebitzinett.

Daburd abet gefhab ez, Dafie doliti=

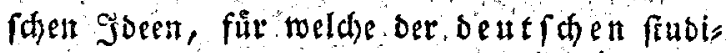

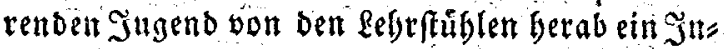
terefie eingefldf wat wa eitte ins Gemuth tiefer

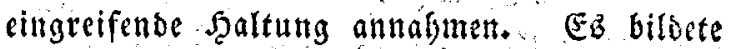
fich uámlid unter ben Joen oon Religion,

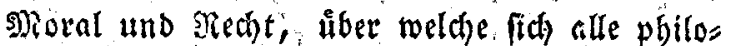
fophifd)en Bortrăge verbreiteten, ein gemeinfa= mes Band, und fo wars bie \$olití ber inga: rirende Bheil eines Snftents, fưt bab bet beut $\{$ d en fubirenden Sugend Sopf und Sers it 2 Infprud fich genommen fand.

(EB wurben baber in Deutfdiand bie politifden Soeen mit eitnem Enthufrasmus uno siner Siefe genäbrt unb gepflegt, wie fonft nirgend. Sie lebten in bem Geift und in bent Şerjen ber Månner, welde mit bober potent=

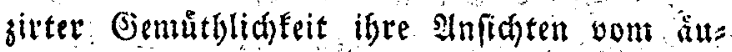
beren Reben geftalteten, uno wurzelten it sen

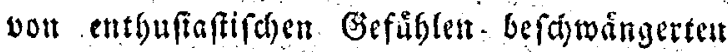
Benuthern cher lettgiertget Jugent.

Diefer politifdse Geift, weldyer anf ben beutfalen feodjidulen lebte, ging aber, bei weitem nicht fo Eraftig inb Gefdiaftaleben b De ut $f$ d) en Bolfz Taft dafur : a bilden und bie Pation felbft fir 


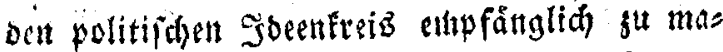
d)ent. Wout ber eitten Esite war ihr Rontur

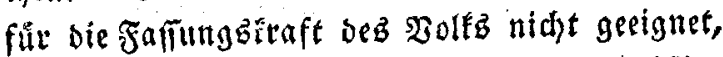
ultb won ber anbern reidnte and ber geiffige Ētoff nidft auz, in biefer rudis indigestaque moles eitne allgemeine Empfánglichteit za verbréten, (EB fand fid gleid)fam cine Niuft, jwifden bett geffeigerten uns erbabenen 2 infids=

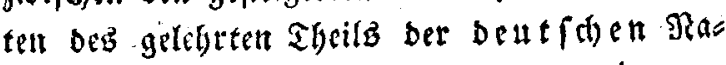
thou riber bew 3neef bes a a

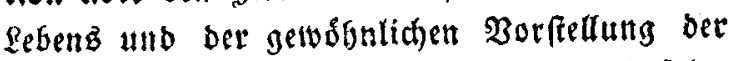
Maffe bez beutidyen Dolfz it biefer Seinfidyt.

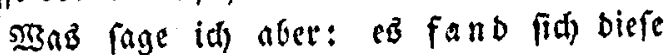
Siluft. Sie bat immex beftanden und befteft nod. - Ea wiffen bie Berfedter ber beuts

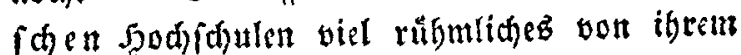
Ginfun auf die getefrete Bilbung Der Jugend, auf ben Fortfdrtte ber wahren 2 Biffenidaft in Deatfdiand und auf bie Entwiferlung bo: Ger Eenialitåt vorgubringen. WBar wird ifuent dies freitig madjen? Siser fant bie Berbienfte verfenten, weld)e bie an bett f̧od)idutlen an= geftellten Gelejuten um bie Altrbildung Der Ierngierigen Sugent und ber SBiffenidpaft von

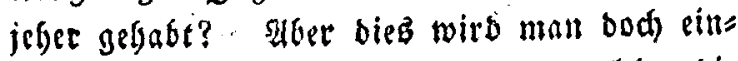

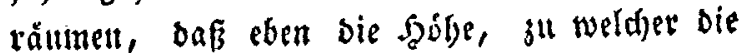
Beiftebaubbildung auf Diefen loben. Sdjulen fid emporgefdywungen, bei weitem ber $\mathfrak{A l} \mathfrak{3}^{3}$

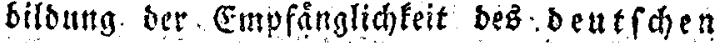
Volfes, fur bas, was den Infprud ben boger miffenfdyafticher sinfidten zufagt, vorgeeift war. Nur lodfer unb faum Gemerfbar war

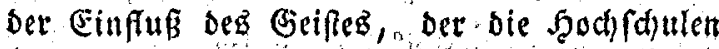
belebte, auf bas wirflide Leben uno Sreiben ber Nation. Jebem, ber nur bas $\mathfrak{\text { Ireiben und }}$

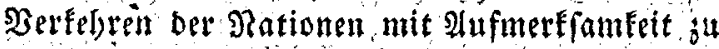

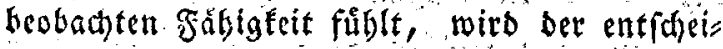
Dende 3 ug in bem Rarafter ber beut fhen

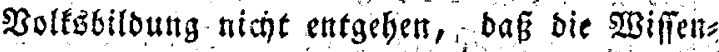
(d)aft bei weitem nidgt fo mit bem Reben bas felbft, wie bei andern Nationen amalgamirt uno eingreifend in bafielbe war. Nirgend fieft ber im wirflid)en \&eben fid Gerumtummelnbe $\mathfrak{B}$ olfatreid mit fold)em Stumpffint, oder ntit beni Srab on Argwobn und sedenflidfeit an bie Joeen unb Entwuirfe, bie ifm von ben isn goenreide leben= Den fispfen gemadit werden; nirgend feeft ber Belebrte in polder fdroffen Entfernung you Gefdăftşeben, als in Deutfalanb, anb nirgend finbet, felbet in den bogeren sreifen Deg politirchen Erbenz, bei Dem praftifdsen Be?

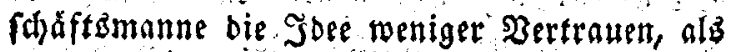
in Deutfiland.

Die boben Sallen batten baber immer iget

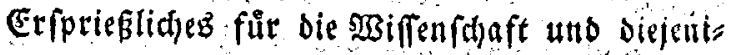
gen, bie fie pflegten, aber fie wirtten_nidgt une 
snittelbat fur ong winflidie feben, anb ibr Einfluf war: nie fo befruditend und belebeno,

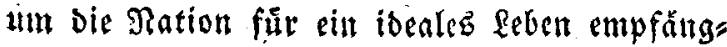
lidi) ju niadset. (Es wat in Deut fdiland inmer eitte flt freettge tht gut faroffe Scheides wand jwifalen bem Siffen unb Dem seben ges jogen, weldye aber blofi burd) bie Drganifation ber Sooufdululen entfanden und erbalten warb.

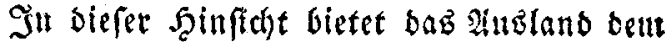
Seobad)ter eine troftlichere Anfid)t bar. Die Sentenj ber fooch fdulen felft bort mit ber bes Doleslebens it engerer 2erbindung. Soee uns Sbat water frthen fid wed)felfeitiger. Dab : Dolf iff empfönglid fin bie boberen Infid)ten ber Gelebrten; alleit bor Gelefrte bertuffedigt audi) immer die subirflidfeit, unb er fapt mur Sas in ber Joee nuf, was ifm die babinrall, fijende Betriebramfeit DeB Bolfez Davbietet.

Wir mirffen ober follten $e$ b uns bod $g e$ ftén, Daß Dab stusland bezfalls eitte Mafte

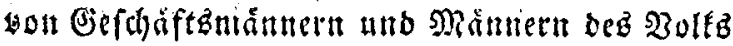
att ber Mation beroorgeben fiebt, die gleidifam in bem Geprăge igrer vielfeitigen bildung eit Riejultak bes rolfalebens find. Sie werben begriffen unb verftanden, uno werftebel unb begreijen. Sit es fo in Deutfalans?

Man mîfte mit ber gróften Blinbheit gefdlagon frill, went man nid) sintäumen

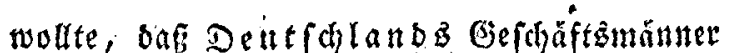
it Şinfid) ber Senntniffe; der Geifteztiefe,

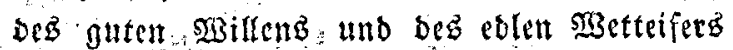
feinen anderes Rationen nadfeben; allein in

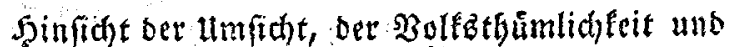
ber ridtigen serwenoung Der Sationalfrnft findan fie ibre Selormeiffer im Iuslande.

Shnen foinmt babei nidjts fu Equldon. EB liegt in bem Drganişmus ber Itnftultell, welthe getroffen find, bie Elttwidelung bet

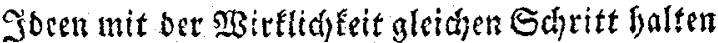

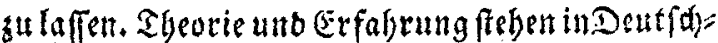
rano in ju weiter Entfernung, uns ber volt

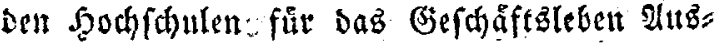
tretende, iff für saffelbe in reinet Beiftebrich tung auf einen Stanoptunt verfeţt, von wels d)eแl aub er von ber SBirflid)feit nid)t in peis nell Inneret ergriffen werden Eann.

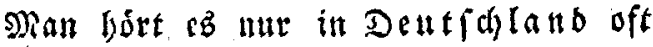
auts bem Siunbe ber auf Scodfdulen gebilde:

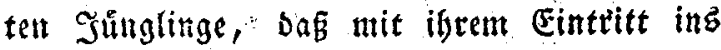
wirklidye leben, fie cine neute Shutle madient mifften, uno ibiten, allet Stoff, Der intten bort jugetommen, ein befawerlider Ballaft

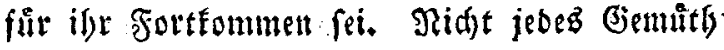
ift gecignet, Den Geift auf Dem Sdiverpunft feftzhalten, vou: welden aus er einer Seitz in Die Jegion bes wiffens und ander Seitz 
in bie bes Sgatbelths fich werliert, Dirfes fels tene Salent bat bie Datur nur cinigen Erlefe= nett verlieben, unb eben bie einfeitige $\mathfrak{A}_{\mathfrak{B}}$ bils bung oder bie Entroidfelung Der ibealen Seite

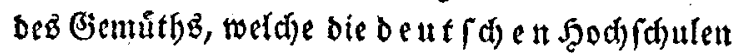
zu bewirfen, vorghiglid geeignet fino, eridiwert

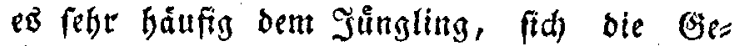
wansbeit für bas thåtige \&eben angueignen, und es bebarf ber junge Mann oft vielleicht elnes lăngeren Zeitraums, um burd) bie Rou= tine fid bem Gefdaftsleben cingt:berlcifen, alg erforberlid) war, feinen Geift für bighere Anfdly= ten empfăngild) gll machent.

Bei biefer Sluft, bie zwifhen der Soee

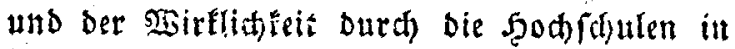
Deutfaland unterfalten wirb, darf es uns baber nicht befremben, onf der Etand, won weldent die Soee aubgebt, eine ganj bon bem Bolle varditedene Grifesriditung auffellte, alb bie Şoee inz wirtlid)e feben liberging, ober alb ein Suterefie fúr bie ben revolutiona: ren Degetenbriten gu Grunbe liegenbin Joeen auf ben Uniorrfitåten wurgelte.

Wenn man genta bas be t the $\mathfrak{Y}$ alf im allgemeinen, wäbreni ber gangen Periobe der frangerfichell gevolution und ber baranf erfolgten 3winghereforft, ju beobaditen Gcs legenfeit batte, wirb man wobl gefteben mifis

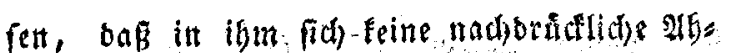
nung oon bem, wab ibm Rotb that uns bes vorfans, aufregte. Bon orm teutfden Voife fann man ez wirklid fagen, bak eb bes. Ginubens vate: nos numerus sumus et fruges. consumere nati. In bobern Birkeln bisłu: tirte man åber politif, auf sod)(d)ulen und in Edriften verbandelte ober reformirte und rewolutionite man bas Gebiet der 2 siffenfdial ten; aber Das 2 olf frano feft, wis ein Felfen, ez fant tidjt aus feiner Scaltung, unb abn: sete nur felten, babi es burd) feine Sitait exft ben Worten feiner Epreder bạ Hebergewidit ju geben vermag.

Die Jegierungen and ibre Deamten, bie (iich bei ber Routine und bem Edlendrian gar behaglich befanden, glaubten recht bod)weife gu bandeln, went fie baz̉ æolf in biefer Rethargie beftátétm, Es feblte ifnen an bem, was ben Deutfden nod fo fidtlich abgebt, an bem Probalitâtablick, ant ber Norberfebungżgabe. Eie fürchteten, bas ఇolf in reiner Apatbie ju ftoren, unb verfolgten bicjenigen ober uns terbrưd fen jeden $\mathfrak{f a u t}$, ber nur in entferntes feen Den Derbadjt erregte; fo etwas fu bes wirten.

und wer waren biejenigen, bie unbefangen und unverfolen bie gefteigerten Infict)ten über 


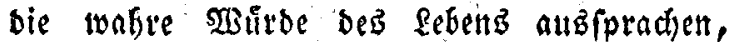
welde fie in ben Gauen beb beut fden $\mathfrak{B a s}$ terlandes fund werben zit laffelt, fich berufen fublten? Grofiftentbeils bie Sünglinge, bie anf ben Sod)fdulen burd) Swort und Sdrift if: rer Sebrer bie Geiftesiricbtang fid anteigneten,

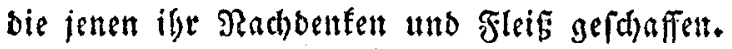
Nit biefem geiftigen Stoff befdwångert, tras ten fie nut in ben Sreis ber Shrigen, beweg:

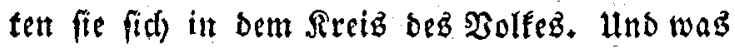
fanden fie bort? Einen Etrmpffint uno sine Unempfănglid)Ecit gegen bie von ifnen aus̉ges forodbenen (sefügle. Hub fie fainen nod leib= (id) weg, wenn man fie nicht mit bent jweis Deutigen Ramen der SReuerer und Revo= Iutionnite belegte.

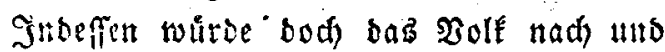

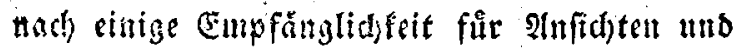
Sboen gewonnen babcu, bie im angencinen

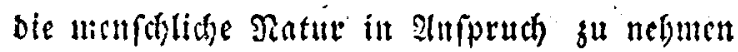
gecignet finb, wenn fie nid)t einem Boben ents trimet waren, too der Solfifitu gleidfam ent: fremost ift, uno wenn bie Soecnreife nidht sinen llanfants bilbete, ber mur einer im Denten gellbten Faffungsfraft angemeffen ift.

Slltf ben Şod)fdulen wat es, wie wir bes

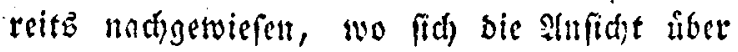

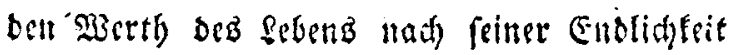

und thendichtert; thad feither gefelliget uns feligen" Natur, butch ben umfdwang, ben bie Begebentyeiten ber Alukentuelt uno bie máditige Entwicfelung eliner Soentolt veranlapt, ges biloet Gatte. Der gange Lmfreiz ber benten= Den Ratur beb Senfben war in Infprath ges nonmen, und nur im Erfaffen cines tranşzen Dentalen Stanopunts fonnte ę gelingen, cin Suterefle für bie iteale Irchiteftonif ber ăt: ficra tutb interen Welt fu gewinimen. S3ie voàre ba zut erwarten getwefen, bab. bie Jing= linge, mit iger son Soeen beraufhten ges mitglididen Whantafie, ber Bolfzmenge vers 'frăblid) werben folntich, und wie founte mait es voun beutfach Bolfe erwarten, dás ez in

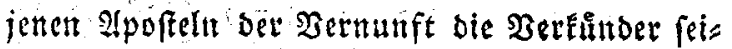
neb gecils fid ofuta follte?

In bicfer Irenmutig, lebte, toie id es nen= "ten módte, bie Iriftofratie beś (Jeifes "nit bem Bolfegeift in Deutfdiand. EB

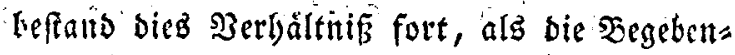

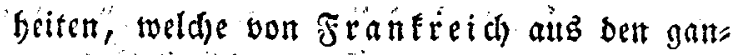
zen Sontinent ummandelten, bie beutfde Geifesarifofraten mit bem empótendfen Druce

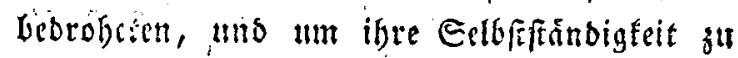
retten; batten fie nut frinen andern Ittaweg, als fich in gebeime semiteen ju werwandeld, 
unt bie Sittel fut etfinten, wie Gewalt mit Getwalt ju vertreiben rei.

Aluf biefe 2 rt entwidelten fich unter ben Geiftesuerwandten Deutfila lands bie man= d)erlei gegeimen Berbirsungen, beren gán zer 3 wed anfánglid) wojgl ber einer jeden ge: Geimen Berbindung gewejen fein mag: Mittel

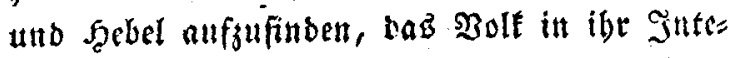
reffe fu fietsen unb es fuit ifre anfiditen ju geswintuet, un in biefer unbewegliden Maffe

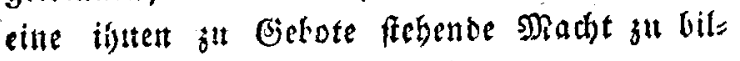
ben, bie ifre Gelbfffándigfeit fu retten vers. mogte.

Matt fâlth bier belt bereits getoobn:

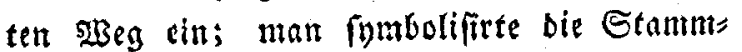
ibeen, bie bem trangzenbenten Eyfteme ber Geiftebarifofratic ful Grunde lagen, um fie bem Bolfe verftăndidaer zu madjen; man per=

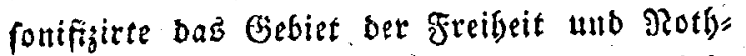
wendigfeit uno bie ifren entprectenden $\$ 6=$ jefte ber Satur und Eittiidfeit, burd) Ebri=

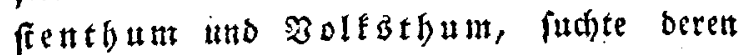
Form im beutfden $3 a t e r l a n b e$ als bie cint zige Hegibe won Deutfhlands beil aufut= ftellen, und fo war bent ellte beutflbe $\mathfrak{T}$ be ofratie gefdaffen, burd relde mant auf

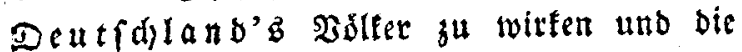
Joer voul untergange fu retten abnete.
So thåtig aud bie Drganlfation biefer Berbindungen betrieben murbe, fo fonnten fie bod nid)t fret und ungebinbert im Folfe ifre Betriebfanteit iben, un fid) eines Einfluffes zu verfidert. Die Regierungen Deut $f \mathrm{c}=$ Ia $\mathfrak{b} \mathfrak{b}$ fowofl, als biejenigen, bie ben Gang ber offentliden 2ngelegenbeiten leiteten, lebtert it Berbăltnifen und 2ierbindungen mit oent Bwitgbern Deutfalands, bie fie verbin, Derten, ifren Gefúblen freien Sauf zu Iaffen, und biejenigen su beguttfigen, bie fth fret willig antoten, ifrem. Sajicfale unb bem ber Deutfden Nation eine andere Ridhtung gu gebert. Snber glaubten fie ood in gewiffer. binfidat politida zu verfabren, diefe Geiftes, ariftofratie nid)tifu-unterbrưden, fonbern fie im $\mathfrak{X e r b o r g e n e n ~ z u ~ D u l d e n , ~ u n ~ f i e ~ i m ~ N o t b f a l f ~}$ serwenden zu fo̊nnen.

Der Entbufiabratus ber Oeiftebariftofratie, Ser vorlåufig in ber Wirflid)feit feine Sraft nid)t vertwenden fonnte, fand nun in ber Ponntafie eitle Duelle friner betriebfamfeit, und fo entwickelten fith im sireife ber beut" fa) Gen Giftezariftofratie fo manderlei

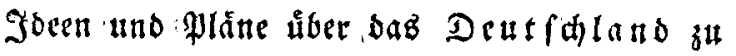
bereitende seil, weld)e, da fie feime Anwens. sung fanden, in sinem bobern Grade abentbeus (rlich) ausgebirdet wurben. IHf biefe.2trt famen 
bent fone grotesfent und farifaturartige Ge=

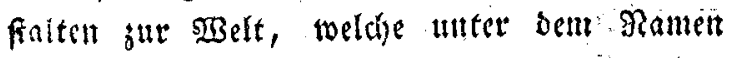
- Deutfdheit fid) reprafentirten, utio von welcher alle die Zerrbilber ansingen, die bem beutfiden Dolfe eben fo itnoerfundidi was ren, alz fie oem ganzen gebildeten Eur opa

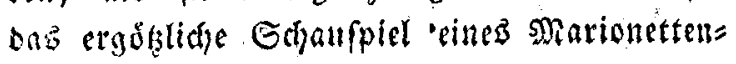
tbeaters Darboten.

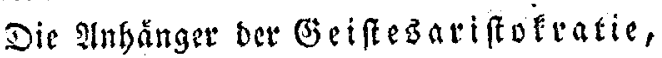

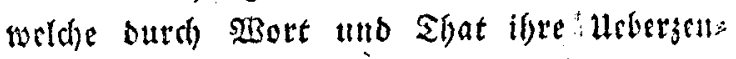
gung manifeftirt batten, fanden ebent in ber Fielte unb lunempiănglichfeit ber Regierungen

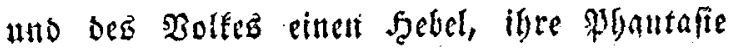
furt tae Sadje ber Deutfabeit in bent

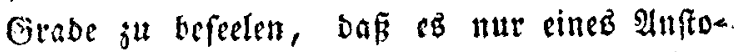
fes besurfte, um ihte Denfart butd) That

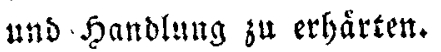

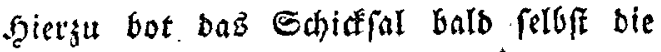
Biclegenfeit, in ber fdnellen wendung, welde bie politijden Ingelegenbriten (E)topa'b, surd Die Sieberlnge ber Jwingherridhaft in

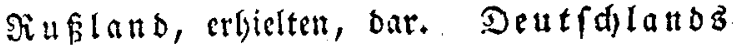
Segierungen ermutbigten fich, faloffin fid bell "torbifden Siegern an, umeinen Sampf ju verfolgen, ber ifhen, ifle Salgftfăndigfeit weieder ju erobern, wnerwartete Plubficht ver= lib5.

Deutfdland'z Bơlfer, erfdiofft, ent: nutbigt unb mancherfeits aud vom Eflaven: finn betăubt, fonnten berolfde Mittel nur zu

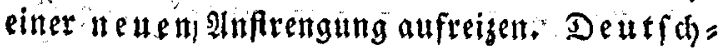
Ia to ítten ein Scebel abjugeben fahten, Den Enthas

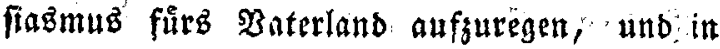
biefer Periode war es, wo ber betferari fofratismus in Deat fd lanb fid gelteno

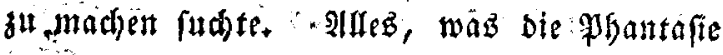
won ber Soee bes Deutfdthums, in bee Periode; wo fie nid)t alz $\mathfrak{Z}$ bat fid) ausf(pred)ett fonnte, gefdniçelt unb gefdnirfelt batte, warb mit einem enthaftaftifden Eifer realiftrt.- EB waren sleidsfant bie: Dpiate, mit weldhen fids Die betitfden Geifezariftofraten gum Simpfe begeifferten, und als mu ber Sieg bon ben Berbindeten unter Den Mauern sont Paris die Freibeit - ben Fontinentalodleert wieber etrungen batte, alb England und

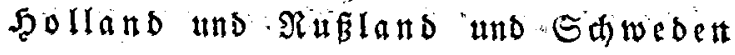
unb Dăltenate und Defterteid, mit De ut $f$ d $\mathfrak{l}$ a $b$, ben Sieg gegen den $g a l=$ Iifden Solvê errungen, fo waren es nun bie

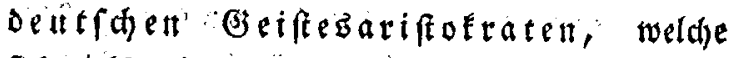
fich als bie -Saupttriebfebern ber Deutfdi= land erwutgenen Freibeit ju adyen berufon shubtent:

Sin-ber Soes, die fie von fich felbfi Gattert; 
gingen fie won bet Jobe ans, baß nicht bie saffen lifermunben, fonbern bie goee nut

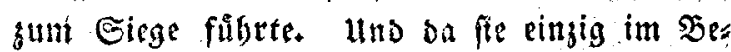
fis berfelben waren, to vermeinen fie aud,

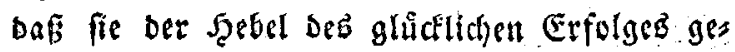
tofert.

Wet wito, wer fant ben eblen beut? fhen Sünglingen oett fdönen Rorbeer'entreis Een, bat fie fid um dic Dpfer erwarben, weide fie dem $\mathfrak{D a t e r l a n d e}$ nit ifrem Slut

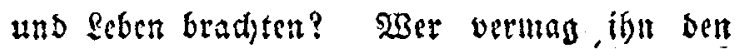
eslen Jưnglingen ju fómalru, beren Oeift; mit ber boben alnficht voll ber Mitrbe bez feligen und gefolligen lebenb genägert, in ber

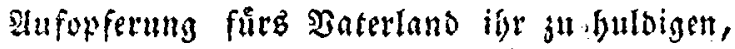
(ich) berufen fithlter? Sidgt in Bewuetfin

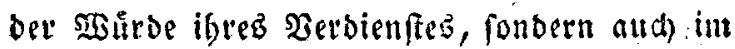
Anerfenmat beffelben von jebru wajuen Deut fben miffen und tocibeti fie igre belobmung

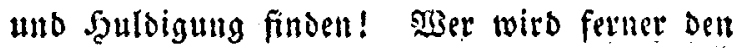
eden beutfhen Mămnern an ben, Sodfdu

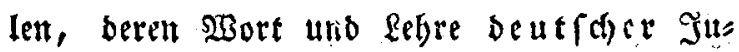
gend Gefubl ju Ibat und Selgerrlidfeit begei fterte, Das fdjóne Berbienft entreiben: wollen,

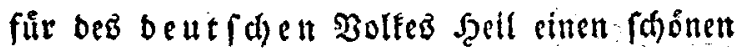
Snamen in ben frudftbringenden Bufen ifrer Sưnglinge verpflangt fll Gaben? - Abev went fie von ber SReinung befafit finb, ong nur
31

ifre Jobett uth bie (Seftulten, bie fid : ourd) fie gebilbet; uns weldie fie in ibree enthufiaftis fojen Sorfterlung von ibrer Shatfraft aubsus. meifeln fucten, obie eigentlident Sebel aller Rationalfraft gebildet uno nur bilben fơnnen, unb wenn fie fogar it ben Grunbsuigen berfels bet, sinen Sanon ber beut fden Ration aufs fubringen; fid berufen glauben, nach welchem fie ifre Dentart in religisfer unb politis fd) $\mathfrak{x}$ Jeinfidt fu mobeln unb gu fixiren baben foll, bann ftellt fie einen Geiftesarift of $\mathfrak{a} a=$ tiş Daniev feiner Gattung getrel geblieben.

Man gebe aber jeget in bent Gauen $\mathfrak{D}$ e $t f d=$ Ianos tmber, Berúdffatige nidht, was in Sdriften uno bon Ratbebern berab in hin fid) tob Deutfatbumb bemonftrirt und Debugirt worben, fondert faffe nur beş Bolfez Irt; beffen Leben in feimen mannigfachen Ges fraltungen ing Iuge. Bewanbter, bothfiniger iff oas Bolf burd ote bittern Erfafintngen genot: ben; aber eben fo einfach; offen, uns cmpfăng= lid)en Gemuttys erblicft man ez nod) wie vormalz, fid) freuent ber Sage, bie oor ifm liegen, uns fufrieden mit ben \$etbăltniffen, bie ibm die Beit und die Begebenbeiten angefdaffen.

- Sprecht cinmal, ifr Geiftezarifto fras te $n$, in biefen Iefendigen $\mathfrak{B}$ olfäfreifen von cus 
ren grofet, weitaughebendent Infidfeit, oon;

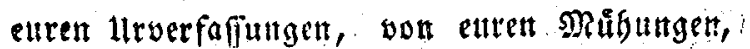
fie wieber ber'juftellen; fellt ifnen tur auf bie sebel, bie ibr it bemienigen, waz: ibt Deutf $t \mathfrak{b}$ um nennt, jufamuen=

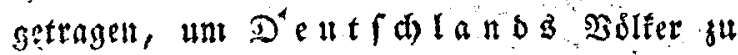
pinem Glauben, ju einer Gitte unb fu ei= uem Rỏner umfuichnffen; ergăblt ibnen wut segenden won bent ewigen Juben, Dem Anti= drift, Der Mirffomteit bes Sireuges. und ber Madjt des Glaubens. Mau. witb eud mit ftieren Blicken :und fummen Etaunet angaf:

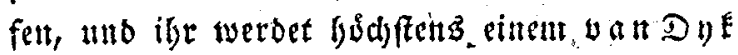

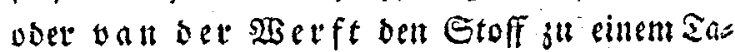
bleaut bergebelt.

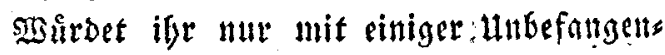
beit "in eures Bolfes sireifen Ginubertreten," fo Eơnnte es euch bein erften Slidfe nid)t ents gehen, dás bie Maffe nid)t fír eure. Anfidjten: unb 2 b fichten fid eigutet Sdjon längf̆ bătten sie Funfen gunden muffen, bie ,eud) in enrer apoftolifden Segeiferung fitr bas seil Deut (d) lat bs entfabren fino. : Sfre fpredjt euth laut uno flaffig genug atts, aber ibre, fefot ilt curer Semperatur fu bod uno bes Bolfes Dhe vermag eä niht, fie ju erfaffel.

Segriffer uno verftanden baben eud bie Negenten uno siadhthaber Der Deutfdien.

lleber
Heber fie fontit ifo atch nidt befagen. Sie

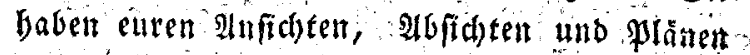
bie Jans treulich geboten; benn fie wånten;

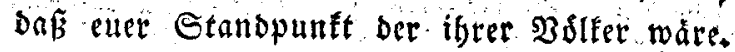
Dab iff ínen von mehreren igrer Beamten vorgefpiegelt worben, bie aud won eben oem

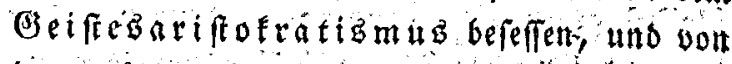

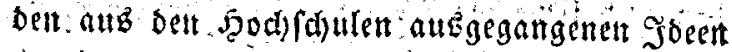
etgriffen fino, Beweife bawn liegen gang est=

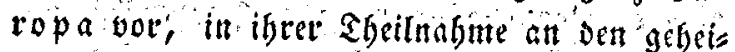
men Berbintungen unb an ben nundorlet mit Feucreifer ausgegangenen Edyopfungen uns. Reformationen, Die im Gebiet Dé Blaubene,

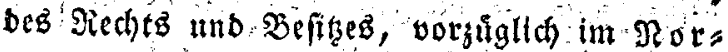
ben Deutfalando, ja Tnge gefóbert fins, unb bies bat eud etmuthigt, bat eud in bem Ginuben an euev 3 ser befarit.

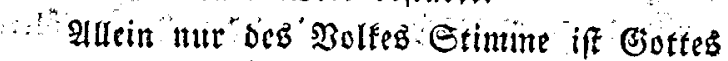
Etimme. Zåglt mir dod anf, was von bem, was ifs Deutfdtb am nennt, bis jeţi, it

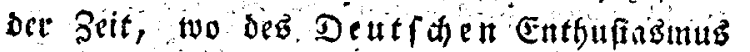
füt fine sBiebergebint in lid)ten flamimen Erante, fid feft in Deutfolano gegrunbet? Das, was man in Deut $f$ d la is umgefhafenes bliaft, iff ein Eigentbum aller $\mathfrak{B}$ slfer geworbent? (EB ift bie Stimme Deg : Meltgeiftez; bie fid)

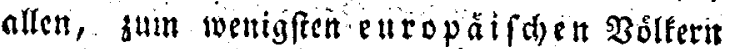

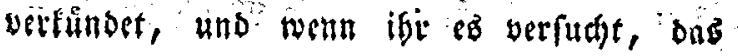




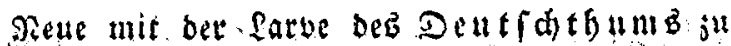
indivioualifiten, fo erfennt jest bas beutfae Dolf bie Nummerei, unb lafit fid) nid)t vou Dem Ġlaban beftechen, Dẩ ể Daz auserlefene $\mathfrak{B o l e ́ t ~ f e i . ~}$

Diefe Apathie, bicfe unempfindlid)feit bez Dolfes gegen cure Geiftesangentungen entges ben cuch feincsiveges. $\quad$ Shr vaboppelt entent Infwanb you Siraft. Sigr bietet, wie ges nandte Partbeigăngev, alle Gattungen oón Dittelt, bie cud) jut Gebute ftelien, auf, um in Dem Dolfacharater ber Deutfden eine

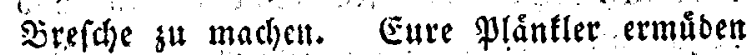
nidjt, bals unit Pfeilen, balo mit Rafeten bie Solear Deut (d) lanob zu unterfalten, uno ibue Slicke auf eud bingubieben. Snbef, bie

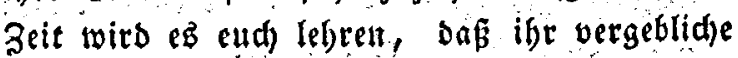

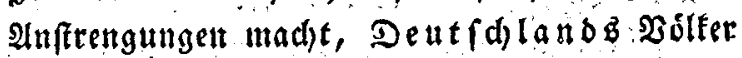

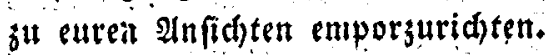

Eure Anfidhten mơgen fuglid alb Etoff fur Bilbung eines politifaen Geiftes zureid)en; aber nur fúr eud, fur eure Geiftedriditung uno Bildung. Wollt ifr aber biefen Beift bem

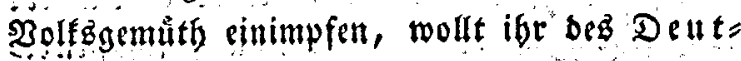
(d)en Bemuth fư ein Siftem von goeen

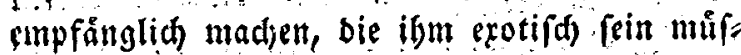

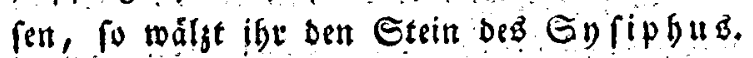
Denn taum glaubt ihe, bie Mafle surds enre geiftigen Mafthinen, Phantafte, Glaube, Be= mutblidfeit, fer ener Allerbeiligftes, bie Deut (abet ; entbufiasmirt und jur beraus (d)enden Betwanberung gebradit zu baben, po febrt fie wieber jurúct in igre Region, um fid von ifeer Erfdióp - ig za erfolen.

$\therefore$ Der wabe politifhe Geift mú im Bolte

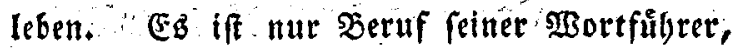
ifn zu beuten und fat leiten. So war uno iff

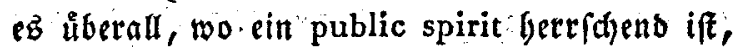
und ba waren alid nur Mănner von Sa)arf blick uno umftat făbig, bas Bolf zu leiter and fu bewegen. Durd)blăttert bie Ge(d)id)te unb -zeigt politifde bewegungen oon cinis ber-bebeutung auf, to das Bolf oder bie Mafle nidht ben Stoff Dagu bergegeber. $\ldots$.

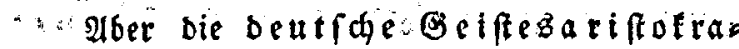
tie, wie beginnt fie ibr Wert? Sie bietet bum Bolfe Stoff und Gorm bar. Eie reidht, wie jener alte Griehe; bem Bolfe ibr $\mathfrak{A}$ age Seabt ifir benn aber ben Geift beb be utfaen

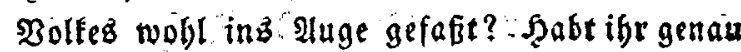
- erwogen, "ob" es burch ea er Auge itt euterem

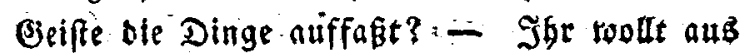
ben Deut (d) Deutfde bilbent. Glaubt ifi benn, bab Die Deatfaen glauben, fie wáren eg nid)t? : Gor wollt ener eud aus altbentfden: Deliquen fufammengeffegts 


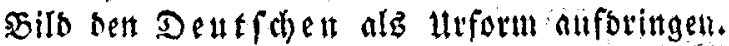

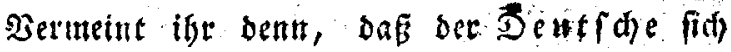

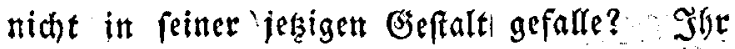
mogt in eurer Sdjreibart, in euten Edjriften, auf euten $\mathfrak{B}$ übnen, itt euren biebid)ten, ihr mọgt in euren Sitten, eutcr Neibung, euren Epielen, Hebungen und Gelagen" bent Deat" (c) en sin Rufter ber Deutfabeit auffellen. Eit panr Saurend Reologen werbet ifr in Deutroland fǜ encl gewinnen, aber bag Bolk, eB wird eure SBeife begaffen, aber ifr nimmer feithe 23 sife opfern.

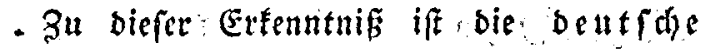

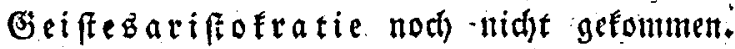

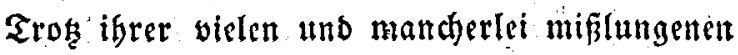
Serfitde; Expeftorationen uno Infintationen auf'z beutfde $\mathfrak{e}$ olf, fant fie, fid nidjt

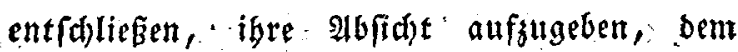

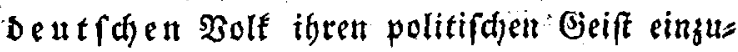
impfen; and es giebt bem unbefangenen $b e d=$ adter wirflidf einen (d)aurerliden Inblick, bie Quinteffens ber beut (d) en Denffraft von einer fixen Ioee befangen $\mathfrak{a} \mathfrak{a}$ feben.

- Die $\mathcal{Q}$ unteffenz der beut(d)en Denfs

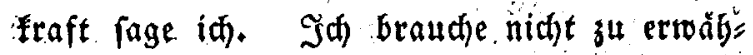
Nen, bas id) barunter bie Maffe ber burd) bie J7od) fdiulet gebilbetent Geifter begreife; baß jut ignen-ふieamte yon ben feltenfen Talenten und profundefent Renteniffer, bie Blithe ber Detitfdẻn J̃ugend, weldie ibren beruf fûr Pflege der wiflenitgaften and ber Geifebbil: bung mit eblem feuereifer berfolgt, uno ens=

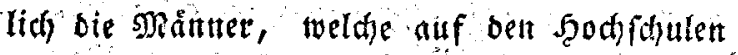
mit rútfichtslofem :Eifer für Entwidelung ber" Beiffer Ieben, geboren. Alfe fapinen fie gleid)= fam von einem Bauberbilbe, bein Şaupte der SReoura gleid), angegogen fu feir; fie ermù ben nidit, ez futbegen und gitaflegen, und crblicken noch immer boffnungsyoul bie Stunbe,

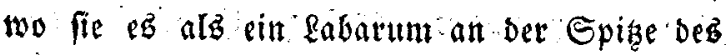

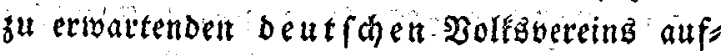
zuffellen vermógent.

- Siăbrend bie beutfde Getfe fratie fid mit biefer sooffnumg gaingelt, tobt wib braupt int Innern biefer fdilagfertigen Metha

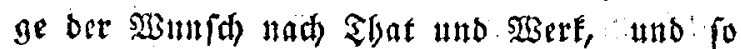

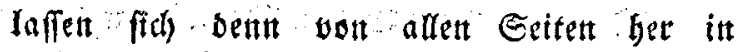
Deutfdland, mie Geiferftimmen, mande

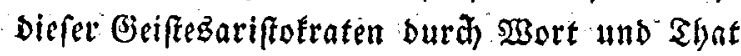
- Dft vernebinen, und ba beift eg benn: ba ift bie Stimme des beatiden politi= (d)en Geifteb.

... Id glaube burch ben bisherigen Socen gang binlănglich erbärtet łแ baben, von wober diefe Etimme fonmt und was es mit ibr für sine Bedentuing bat. Gern wifl id cinråument, 
baß ez politifde Geifter it Deutidland giebt, politifder, alz fie trgenbwo angetroffen werben. Sie finb $e$ and, bie mandent Gremoling irre fübrten, unb thm ben Glanben nuforangen, ons foldye abnorme Geiffesindivi= bualitåt bez̉ beutia en $\mathfrak{D o l f e z ~ g e m e i n f a m e s ~}$ Erbgut geworben, unb feiner bat fid vor Eurs zem ro entfhicoen ưberilt baruber ausgefpros den, als bet feer von Stourdja.

Man bat biefen ferrn bon. Stour $r a$

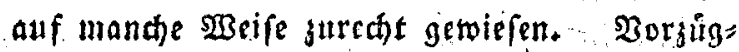
(iid) baben mebrere Etimmgeber bez ofuts fdett (seifesariftotatiomub fid) bert= fen füblen múfen, gegen ibn mit Näd)bruck aufiutreten, inbent man betennen mus; bas er ifnen fu nabe trat, unb ibnen; 9 bfid)ten .unterfdjob, won benen fie gewif entfernt fein Dirften.

Wiggen auds unter Den (Beiftebariftofratent megrere Indiviouen feit, weldse von eigen= nutbigen Iriebfeoern geleitet werben, fo wat es bod immer ubereilt von einem fernen bes obadifer, foldye 2lbfidten ber Befammtjabl un= tergufdieben. Der beutfde Geiftesarifte fratizuus bat getwiés ben reinften unb ebels fen lirfprung, ben. je cin Arifotratiomus batte. Jerr von Stounda batte erft biefent gennt verfolgen follen; oann wurber er vemo,

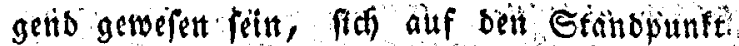
beffelbett emporgulebet, and fid bin gewis, er warbe gans andere sefultate befomment baben.

Bon Dem Gefidfezunft, bon werden aus

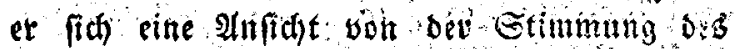

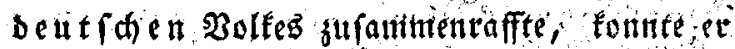

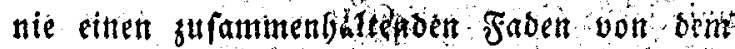
Gang ber zeitigen Geifestedtutug in $D$ e ut $f$ dy land auffaffen. ". Setr son Etourbia ver fennt gang Den edlen zwed bór Geifegarifto" fraten, bem deutfden 2olfe eiven poltit:

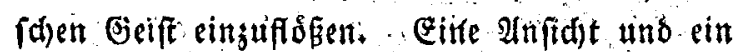
Streben, Dab ifren Ederfint und ifre Yater

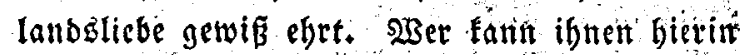
einet Irrtbum zurednen? Aber bas ift ifr gemeinfamer Grrthum, safi fie es nidjt erfents nen unb nad fo mandietle girufung nidit cin: feben wollet, oás biefer poltifche Geift feine Entpfánglidfeit brim o e ut $t$ d en Polfe finden fann und fold, cinen $\mathfrak{I} a f t$, ben nan bodj bei

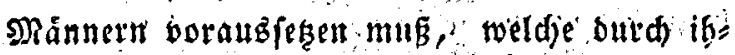
ren Geift eiven folden bohen Stanopunft fur ibre Anfidt voln politifien 'Reben' ex rungen.

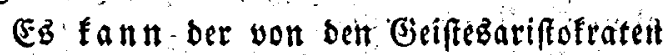
entwidelte politifde Beift in Deatfalano Ecimen feften Fur faffer, weil ein 20 lf bot 


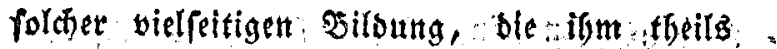

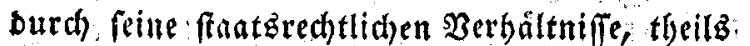
burd) ben auf: es influirenben Weltgeift fuges fommen, fid) (d)werlid) gut ciner Form bequement Duirfte, bie ber univerfelten Empfănglidefeit nid)t zufprid)t. Run iff aber bie Sendeng bes Dentfdthats: eben bafin getichtet, jene

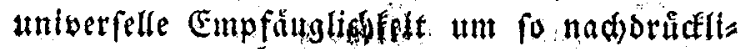

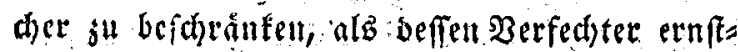

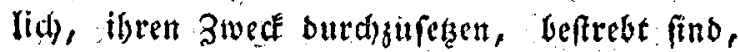

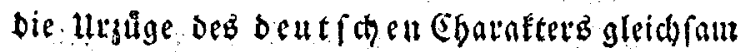
fut reffauriren. $\quad \cdots \quad \therefore, \cdots$ .... Es: fdreint aber, nadh bem alfgemeinen Gang ber Rationaltildung, eben Die. Natue wabint: ju arbeiten, bickirjugge ber. veridiedenen

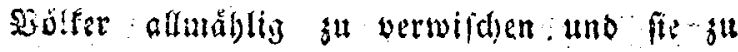

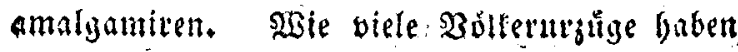
in Dem grofern Pattorama Der Yólforplypfios gnomie nidjt ifre Eigenthümlid) Eeit vetioren,

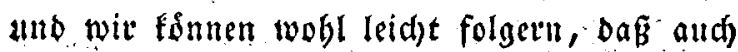
nod) bie beftegenden in. Der Folge Der : Jeiten babinfdwinden wardett. Dem, beut rafen Wolfe erging $\mathfrak{e B}^{3}$, wie allen Pationen, und e⿱ ift nidgt eingufegen, wab es babei getwùne, wemit Der Serfud der Deutfdhthumbers efjer $\mathfrak{e} \mathfrak{r}$ gelingen foffer.

5. Es if wabr, jedes 2 olf bat feinen cigens thinliden Charafter, feine befonberen: Sitten.
Šbes geidfnet fid burd eigene Epradje titto

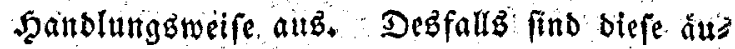
Beren und gufålligen Attribate nidht nothtwen Dige Beoingungeit, einer bebeutenben ! Mafie

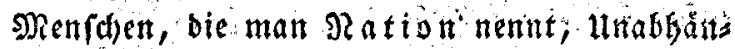

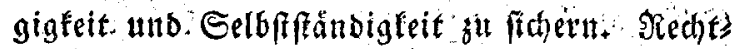
lide befinnungen, aubbaunde betrieb jamert und Gefese, wela de biefen motalifaen und phyfifdea confift aufred) erfalter; bae fino bie twabren Pfeiler allet Mationalitåt. Sod glaube bod wohl, baß, feitbem uns bas Schidfal in ber neuen welt in ben oeteis nigten Staaten, eitr. Excmplar vou reinct foldijen Nationalităt aufgeferlt, bie al te Solte

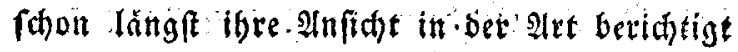
baben follte:

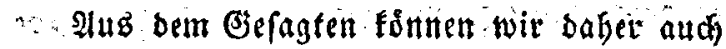
bie pragmatirche Folgerung jeflen: Dafis bem

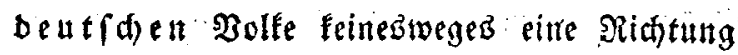
aufgebrungen werben foll, wie bie 3eregrer bez

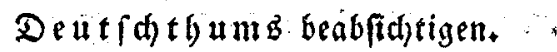

Diefe welthiforifchen 'und pragmatifaen Momente überfegen aber bie beatfden Beis ftezaciftofraten, oder wollent fie fum wenigfert alş unerbeblich gegen ibre sinfit)ten gelten Iafs ren.:- Alte Ereignifie, alle Folgen, welthe ăus bem Untergange ber zwingherridgoft in bem

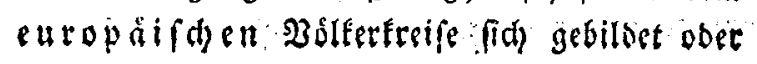




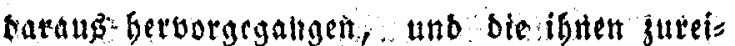
d)ende Minfe fein follten, baf ibr Seftreber cine vergebliche Inftengung iff, bleiten woit

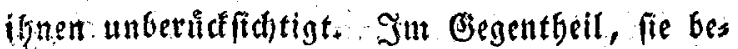
tracten fie als fo sid Excitamente, die bab Erfictial ibuch auffeflt, um ibre sinftengung

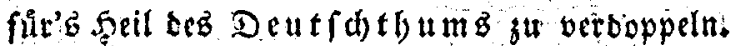
und was ift biersoul bas Refultat?

Da nun die Geiffezariftofraten fehen, bafi fie : nach aufent bith wenig gt wirken uno ju beivirfen bermógelt, daf fle vou allen Geiten ritten ulzertwatteten sisiderfand finden ober zu finden abtuen, fo congentriten fie fid nut auf ibre Gemuthlidbteit. Sie berdionăngern fie mit ben Soeen, welde fie gu vertwirflid)en fdont tuăbuten. Sie erbliden nut ibre seit in bent

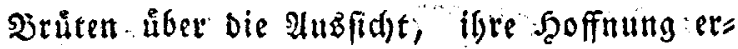
füllt iu fefen, und glauben fid für igre Ees ligfeit vorguberciten; wenn fie, beraufd von foldgen Gefulglen, die Zcit verleben.

Sn diefer vergweifelten Stimmung ift bet

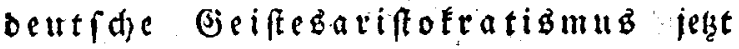
verfunten. Edion ber sBiberfand, ben ex bet feinem Bolfe findet, muß ibn entrůften, um

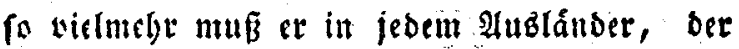
ibm Seinderniffe veranlafit oder bas be ut Dolf oafur ftimme, Deft beftigften Beguer fid) centen, ber nid)tó weniger bezweckt, alz nuf bet Trummern bes beutfden Zaterlanbes feinen Triumph fu feiert.

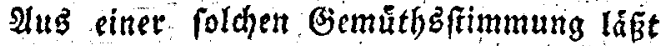
(fid) mur bas tragifde Edictial, bas gang Eu= ropa mit Sd)atbern erfúlte, erflaren, Das unter ben hăben eines som beutfdent

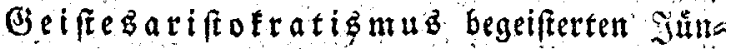
gers bem Sanne bereitet pard, ber mit bem.

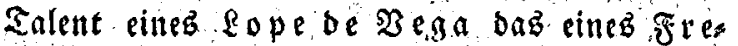
ron vereinigte: id meine, $\mathscr{H} \mathfrak{a} \mathfrak{a} \mathfrak{f}$ von

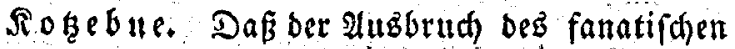
Eifers eincr in Deutfalanog Saoof fit allmáblig gebildeten sartbei an biefem ausges zeidnetent Manne fid) erbátete, fictiert fir ins mer in ben Annglen ber s)enidhbet, biefer gang eigenen fid entwidelnben Geifes ridtung cine pragmatifde Bebeutfamét." \$it Dem oauernben Andenten, an eiten frudibaren Geif, ber ein Dpfer berfelben warb, wirs fie aud in oer beutfden Sulturgefdidste eine ifr Dauernde Stelle ergalten, uno wir glauben, Daß die von unz befolgte Sethode, Deren.ur= fprung unb Entwicfelung barjuftellen, it bie=

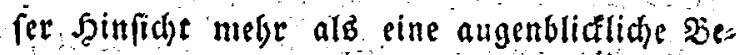
ridffidtigung verdienen wird.

Inbem toir aber bie fúr bas Deutf - thum begeifferte Stimmung als eill, burd

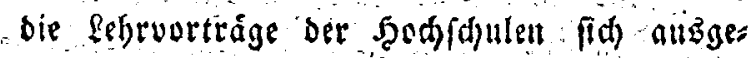


Gilbetes s)inanta entwidelt, uno eg als ben Grathoton bes politifate Getiteb in Detithas

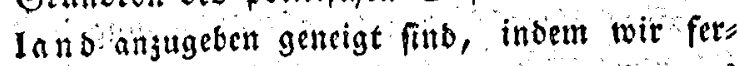
ner biefen politifasen Geif, peitteg uriprungs ans fetier Entmidkelung gemáb, soriuglid in

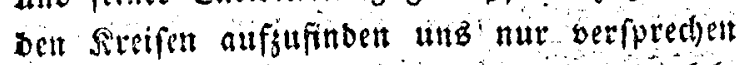
bitfert, bie mit jenen Geiftesanftalten in lets

baftefen Berfegr geftanoen unb nod fefert,

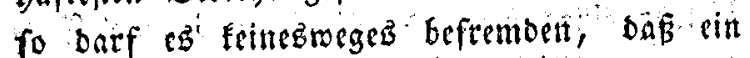

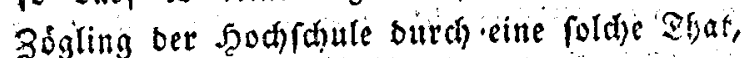
wie bte Ernotsung Robebue's, ben Ein =

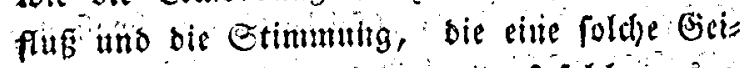
fesciddtung veranlafén inag, anf folde ausges zeid)nete siseife manifeftute.

Entguftaften fuir ein Syftem, für cine Micinung find immer bem Boben entwadsen, wo fit cin Enften, cine Dieinung ans gebilbet. serna wir. Glofi in ber nettern Gefchid)te uns

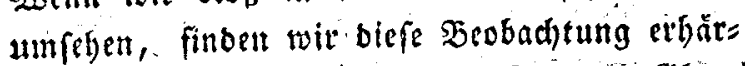

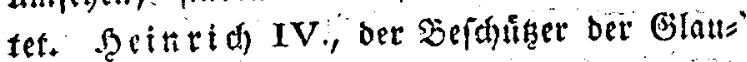
benofreigeit, ward cill Dpfer oeb Fnnatiomus eintes jullgen Geifflidyen; Niarat, ber politis fde Partbeiganger, fiel unter ben Etretiden ciner freien Republifanerin, und bes Sodnigz son Englanb leben-ift oft Den morostifden Anfalle:t cihes feiner fpleenfichtigen lintertbas

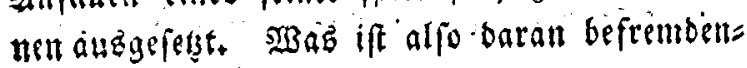

Deş, Dấ So

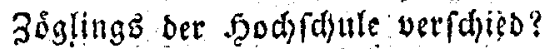

Desffalle lafit fid aber teinebweges folgern, bas bie sodyrhulen felbft zu einem fold)en $\widetilde{F} a$ matisumb it ifren Sreifen bie Gemuther fit fteigern, die eingige Beranlafintug wăren,.. Mian wirbe bier ben f̧odjfajulen in eben bem Gras

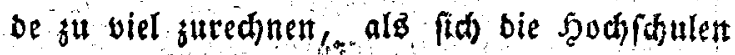

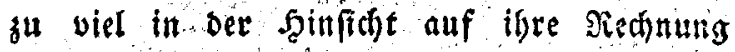
fdreiben, went fie bie Infidht auffellen, $\quad$ a fie cinjig und alfein bie wahren Sofỏnberer, Reftauratoren uns sifeger ber wahen . Niffent= (d)aft in Deutfhlano reten. Wir finden

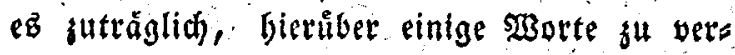
lieren.

Dab mafre $\mathfrak{B} i f \mathfrak{l}$, dab, wab ber Geift mit feiner Sraft auffellt, ift Dab ßefultat eis nes, aub fith feloft fich entwicklnden Probues teb. Go unterifheibet fich von bem \$iffen, Dabiourd Mittbilung, Erfabrung und Etus Dium erlangt wirs. Es twirb niemand firt flt beljaupten vermefien, oẩ eine geniale Jocen, reibe das Produft eincr fold)en Mittheiluttg ift, fonbern bỏdftens bie Beranlallung bu bers frlben. Wer burfte bebauptel, bie Fortfdritte

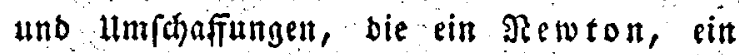
Eeibnig, ein Nouffeau, ein Frantio, sin Pinne, cin $\mathfrak{B}$ infelmann, ein Gơthe 
unb ein sant in ben nattdertei Bebietch DeB SBifiens veranlafiten, beit Mittheilungen, bie ibnen alf ben feod) fhulen geworden, ige - Dapein fll veroanfeil babell. Die Scodffaulen bieten bem Geift nid)t meb́r als bie Werfzenge bar, woourd) ber geniale Mench reine Rirăfte gu fanbbaben lernt. Es fino die Sebrjabre, bie Der Cisift, wie ber Sirper, zur llebung verfeben mus. Wâten bie Mittheilungen, bic auf ben hod)fdulen ben gunglingen verlieben werben, von joldjer allgemeinen prosuftivat sirfframfut, als es jene genialen sopfe fino, fo wurbe ein gans anberes sefultat aus ifnen brroorgebet müfen.

Benau genommen, berfaffen mefr benn oveiviertsl ber Jinglinge bie Şod)fdule, blob nit folber Renntniffen angaeruftet uno fo

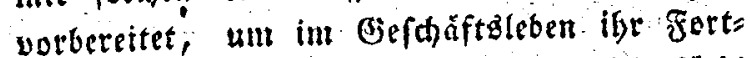
fommen ju finben. Die (Empfăngliợteit für's walfre wiffen, uno ber Eifer fút ben fort: fdrtt besfelben, finb Talente, von benen fie feine Abnung baben, und es geht im gerodge Iiden leber fo roeit, bafi nan Enjeto, bie ci= nen Beiftesjidwung Der Art verrathen, fire Ans: malien in ben Difaferien betradjet. Der Groffi= theil ber MRaffe ber Bilbung alfo, bie bon ben

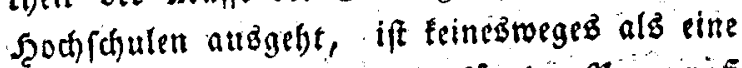
Brofie gu betraditen, roldhe fúr ben progres
Der Noiffenfdaft in Nedjung gebradst wetoen tant?:

Die wabren genialen Ro̊pfe bilben erft ifr Salent eigentlid nạd) überftandenen febrjabren, rei es in Gefdăftaleben felbft, ober währenb Dez Serufezs oem fie fid als sefrer wiomen. - Die lebrer Der Szodjfaulen úberfdåtzen ge. wifi ibrent Einflü, wenn fie alle won fid) glaus ben, bá bures ifre Mittbeilung Dem Progrez̈ bes $\mathfrak{B} i f e n$ ẹ eit eigenthúmlideb fundament gelegt ift. Dex: Siffenfhaft mo̊gen fie eine Fortbauer fidjern, aber nidit cinen Pro geen bem Wiffen.

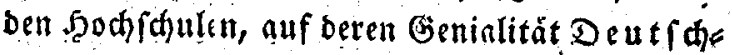
lanb ftols su fein berechtigt iff, baben alle ibrer Wirffamfeit ein weiteres ziel gefeb̨t, alş ben, bor einem Sireife blof Iernbegieriger Jüng linge fid) alisjupredien.

(F) ift baber immer cine mit etwas quvies ler Buverftht ausigefprochene Deinung mehre rer 3erebrer ber J̧odj(dulen, wẹn fie foldje alß bie eingigen Yifleger, Ertweiterer bes $\mathfrak{B i f}$ fens betrachten, uno baß fie Junfitute wåten,

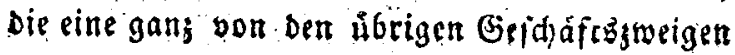
Deş Etantes verfdieden organifute Nerfafo fung uno Befebgebung baben mủsten, woburd) ifrer Euprioritat gleidfam das Eirgel aufge orût meroen foll. 


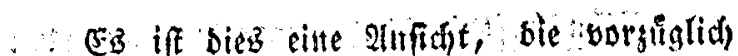
feit furzell mit viclem Eifer verfochten wird,

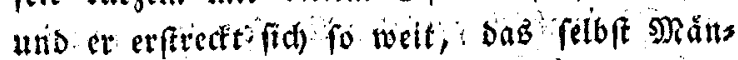
net, jueldie ben Refriaben der Sdaule längft entgangen, fener Anfid)t saburd) ? cine gobe Setebrung zut zollen glauben, wenn fie alliăbr (id) ourch cin fogennnteb Meeting bie Frins vtrung an ibr unioerfitåtaleben zul feiern, iffentlich verfinoen.

Diefe bohe, metreber weibiger jeft dfs

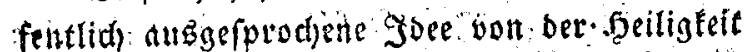
ber" 50 d) faturn, giebt uns efnen bebeutenden Subion oem politidaen Beift Deut $\{$ d) linos, und evbårtet, was wir eben ausges

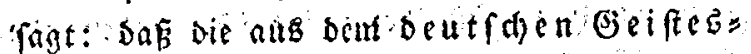
arifoeratismuz beroorgegangene Geiftebs riditung fein Gennoten iff, Dieb bietet uns aber Den bebeutenden Moment dar, baf bee

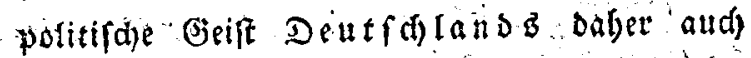
bon bor Ratur uno sit ift, um in elinem be" rahrånftett Sircio, aber Ecineşweges in bét Ges mưthlid)feit ber Nation ju tourgelit.

... S. Sicraub bilbet fid aber eigentlid) bie $\mathfrak{V e t s}$ rajicocubeit in. Der Nationalitát Des Deat”

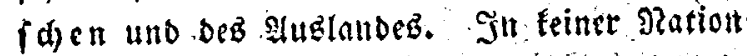
iff bie Goee von bem Gemüth fo getrentit, wie in ber ofuthen., Die gation ift eigentlia) getbeilt zwifden Geift und Sim. ... Sies be= wirft wirft in ifrem-politifhen unb geiftigen Reben eine Silft, bie (d)werlid) fo balo beretiget. werben Eann. Die, bie Beiftebfultur, $\mathfrak{j}$ pfies geit, Berufenen, bnben ifren Fond aub Dem univerfalen Fortidritt. Der Wiffenfhaften auf

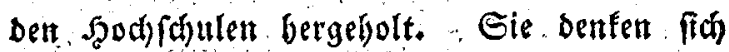

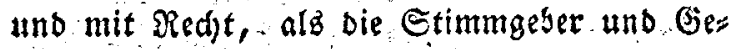
feßzgeber von dem Gefübl= und Joenfueis des

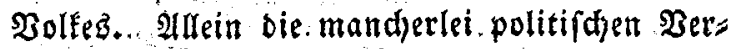
băltuiffe bez. oeut $\{$ d en. Bolfeb baben ebs ver

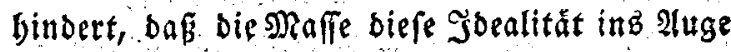
fafte, und fie blieb baher in ifrer Geiftestbas tibleit zurud

Frok ber manderlet Beriuche, weldje bie Gelebrten ober Geiftesariftofraten veranlabten, Das Bolf ju fid emporjubeben, ober fid mit ifm: fu.familiarifiren, fo Gat man bennod bis jeht Eeinen bedeutenden Erfolg gefehett. Dett in, bie Alugen fpringendfen şeleg geben bieju Die mandberlei Beftrebungen ber beutiden

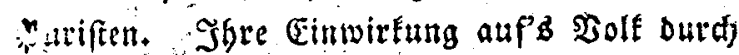

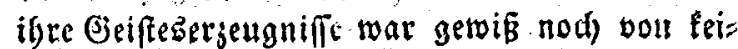
uen erfolgridien (Einflus, unb buirfte es aud fhiscrlia) fo bald werden, da fie unter fí felbft nod) feinell bebentfamen Drganismus entwickelt, Mau Finnte beinabe won iguen faSen, waş Fontenell von den $\mathfrak{B}$ a 
$5 \mathbf{r}$

Bert: "\$or bebauptet, baf fie fid untereinan: ber verfteben. Id) glaube es̉ ticht."

Die Drationalitåt, weld)e bura bent bon ben Scodfd)ulen und Geiftebariftofraten $\Pi$ (d) entwidfelnben politiftyen Geift ben $D$ ent fiden atgebilibet werben folf; ift baher gletdfaim cin Fremoling in $\mathfrak{B o l t e}$ Er will fich ăber bab 2ole etheben unb ifm bie Riditung geben; ftatt bas bie won ben Geiftesariftofraten ober Selebrten im 2 aslanbe angenommene InbibiDunlitât, erfic ifr Fortfommen finden fanit,.

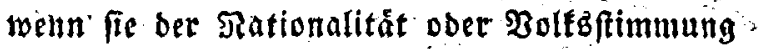
fit) Jingiebt, bie immer bas uebergewitht ůber. bie Minoritåt fu belanuten fid vorbebălt.

Sa) zăble diefe wandelbare Nationalităt

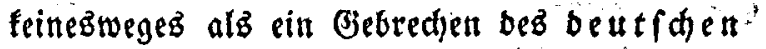
श̧olfes auf, fonbern (d) betrad)te fie vielmetre als einen von ber- Ratur ifm eigentbuimlia vorzugzweife verliebenen Raraftergug.

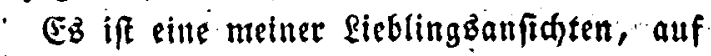
Die (d) aud) fajon in mebreren meiner Sdyrifs

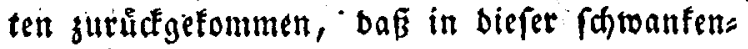
beñ Nationalitåt ber. Deut $f$ d) n nur eigent:

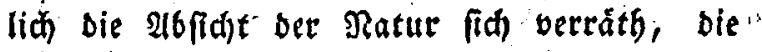
Diut fung aller Rationalitåt fu verwenoen, benn' id frage: weld)e Gefere Gat bie Satur att: " geftellt, bie eine jebe Nation verpflid)ten ten, frenge, auf thre Rationalitat fu balten? Singegent finde id) aber, wenn ith oie Annalen ber: Yolfer burdagebe, DaÉ eben Die vormals

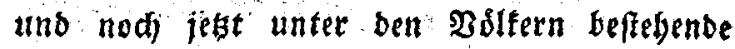
Rationalităt, ber Entwidfelung ber Joee einer Menfhbeit, bie ber men/fliden Ratur burd bie Dernunft offenbart toirb, im Wege fieft."

Zivar wollen bie Inbănger und $\mathfrak{B}$ erfed)ter ber Rationalitåt benen, weldye Der Entmictes. lung ber Joee piner Menfhheit anbången, nid)t oolle Geredjtigfeit wiberfabren laffer. Sie berboftren infere Infidjt, und erblicten barin bie Geburt eines bobenlopen Rosmopolts tiżmus, Der baš ofnebiez befd)rănfte Gefúb! Der menraliden Ratur in sine gebaltlore Gas= art verfluctigen burfte; roburdh bit edelften Bicfuble im Menfden endlich ibre Sebertung verlieren mullien.

- Jubeffen, ifinen fant man arwebert: Jbeen, Begriffe, Gefulble bedirfen teines Ronturs ano felrier Geftaltung, und eb ift immer sin $a$ thropomorplizmus, fie in Oeftalt, Slima ober Boden, -alt Eitte und fartommen, an Spradhe und gebensedet fe ju fefteln. ano was enthált eure Nationalltăt fur andere Nttribute? Des Geilige Blaube; ber getweibete Boben und dic Eitte ber throrbern, finb einig unb afleit 
bie Flosfellt, outh welde ifre ben Spielraum ber Gefible auf cinen : beengten sireib cins

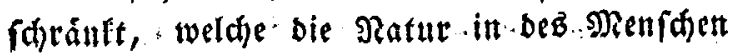

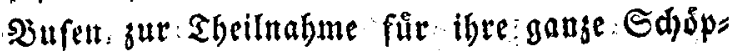
fung binverpflangt.

Rein! befeeligend unb eingig befeeligenb ift

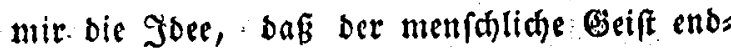
lid babin entwidelt werben wirb, bie Buirbe Der gefeggebenden Bernunft alb fein eigenthŭm: Iidyftes Erbtheil zu ad)ten, unb fie alsz ben Mieifter feiner $\mathfrak{B}$ ilfübr anquerfennen. uno befeeligender whirbe mir ber Beitpunft bann fein, wenn id). Die worte beż beut faefen Dichters:

"Seio um[d)lungen Sillionen!

Diefen Siuß Der ganjen welt!

Brúber - ŭber'm Sterneñuet

Má ein' lieber Dater robnen: "

zucrfian Deut $(\mathfrak{d})$ Iand in Der. Wabrheit aubz jubringen bermogte.

Diefe meine Anfint liber sie beftimmung Deb̈: Deut fd) en : Dolfes twird - mir aud) Durd mandse 3lige erfărtet, Die bie. Berfafinth und Die vorgegangene Bieiftesentwicfelung: $D \in u t(d)=$ Ianb barbietet.

Dbgleidj bie Sprade ein allgemeineb ßanb unter ben: Deut $f$ d)en bilbet, weldes uns in

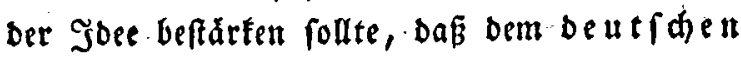
Bolfe eine in fid) felbft fortfádeitende..und volle

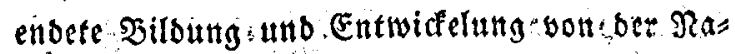
tur angewiefen: fei, fo giebt es boch verfdis: bette anbere Derbáltniffe in ber lage ber Dent fden, bie uns wieber bie Sbee nuforin= get, bafi Deut falanb baz Receptaculum fi: ner vielfeitigen Sultur, ben Bollern auffuftel= len, beftimmt ift.

- SBie licke fid fonft in ber gangen politis fden. Derfafung Dent (d) I and b, wold be bie intere Rraft ber Sation gleidyfam firh feloff Plete glebt oder. fie in bem Gange ermudender. segociationen gleich) fam vergenben, lơfit, eit : teleologifdjer punt auffaffen? : uno wie wứtbe fonft ber, it biefen, won ber satur gletd) fam in ben Sd)oós beutfder : Nation bingelegten burd) gegenarbeitenbe Intereffen, fith entwidelts be Siater einer perpetuirliden Găbrung, jene in De ut $(d)$ la n b ientfanbene und - nod) beftelsen=

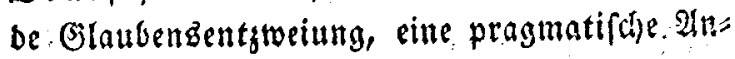
find getodibent to̊nnen?

Rur eingig und allein in : der Berfolgung ber : Soce: Deutfdland fei won bem Sald" fal berufen, , fû́r bie weithifforifhe Alubits

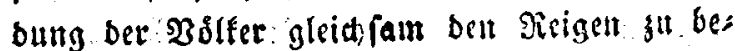

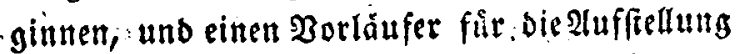

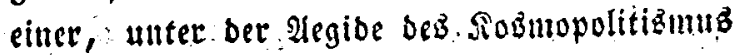

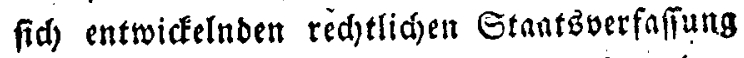

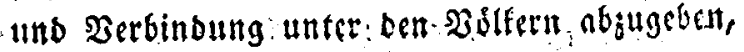


fant $\mathfrak{D}$ eutfdranb bent benfenbeth Politifer ein erfolgreides Refultat in :feiner feitigen Giffaltung auffellen.

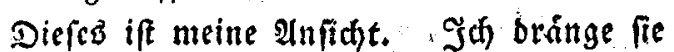
feinem auf. Id) barf aber bingufugen, baßs baż ganfe benebnen bez bentfden Dolfez in biefer, alle politifden Berbăltniffe bewegen: ben zeit, fid in bet Art fignalifit, bafi matt wobl folgern barf: feine gange Enipfinbungs: und f̧andunganeife fei fưr jene to fd) Geiftebrid)tung von einer bơhern Mad) (tt Fefalag genommen.

Diefe Altfid t tann feineštoegez̉ Geifállig von ber partbel aufgenommen werben, weldae bem

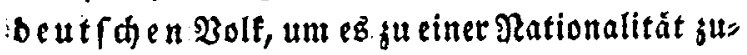
růfjuleiten, ein rogenannteb $D$ eut $f$ d) $t \mathfrak{b} u$ ans fufdaffen, beftebt ift. Wुir baben ben Sarafter - ber SBortfibuer unb Sünger biefer Partbei nidjt alfein in bem Sauf unferer Joeen fennen gelerut, fonbern aud ben urfprung unb bie Entwides lung ifrer Anfidten bem Sefer vor Augen ge= regt. Sie funb, wenn wir fie mit wenigent Worten farafterifien follen, bie Reprăfentan= ten eines beutfden Seiftezariftotratizmuz, ber feinen politifaben Geift füt einen, ber oeuts

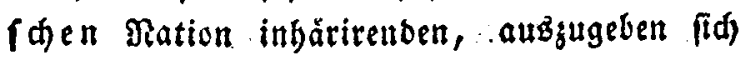
beftrebt.

Dấ fie aber, wenigftenz in einem bebews tenben Ebeile Deutfalanbs, uorguglid in nórblid)en, fid in einem gerwiffen Brape geltend fil madien bermodte, wird uns feiness wege ble lleberzeugung anforingen, bẩ fie fid

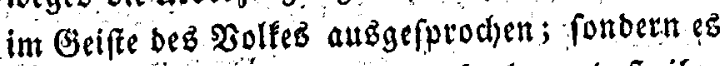
wiro nur ein Allegat mefr afgeten, daß̧ íle Denfart eine frudat bes Geiftesariftotratismus ift, weil im noovoliden Deutid)land un= begneifelt die Geiftesfultur ciner felbftfonnoigen Entivictung worbehalten ift.

. Die Veronlaffung bierła $\mathfrak{g a b}: \mathfrak{m}$ nơroli= d) Dentalanb, und sorguglict in Dem preafifhen und einigen mit ifm auf glei d)er linie oer (Seiftesbiloung fefgenben Staa teit, bie unter einer nambaften $\mathfrak{I} \mathfrak{a} a \mathfrak{b l}$ von ba: ben Staatabeanten berbreitete beutfhe Gej

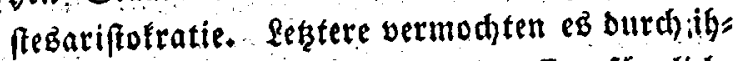
rén Einfü̧, ber Siegierung cine Empfä́nglid)= feit fár ibre sinfichten einfufléfen, uno brad) ten ez babin, baf oaburd mebrere in isrem Geift organifirte sffentlicte Anftalten jur 2 ers

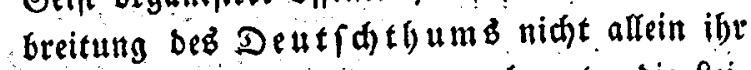
Dafein erbielten, condern auds unter bie $\mathfrak{E}:=$

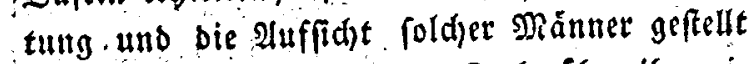
wurben, bie mit leib und Esele liber inte ci= gentlidje Eendeng wadten.

Je mebr fid bie eigentlide form uns (bes ftalt biefer Juftitute entwiafelte, uno ber it 
iftert fid regettbe Obeiftaitsfpead, befto enfo fheibender fpritgt aber ber Sontraft ferwor,

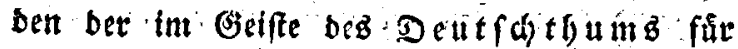

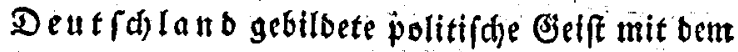
witflich befregenben hat.

Woir braudset ben seitgenofien nidht voigh fúbert, mit weldjem Eifer bießartbei ber be tt t= (d) en Beifesariftofraten fith anftengte, ber Bes

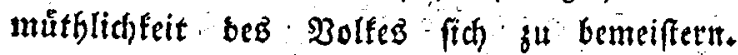
Ifte \$gilofophett, ifre Religionzlegrer; ifre Ergieber, igre Naturforfder, ja ibre Alerzte, traten in eitten nod) nie Statt gefunbenent Bersint: Sie bradten : Materialient uto Mias fdineti zufammen, wie fie bie Yolfabilonet

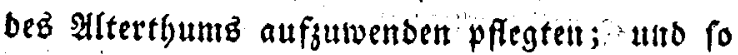

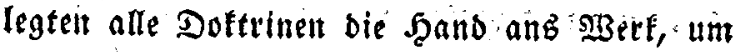

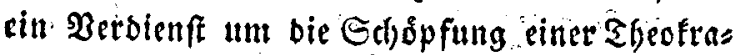
tie zu baben, bie fie mit bem Ramen Dêt thum belegent.

Die preusifde segiêrung bertratte bies fem Eifer; fie reid)te grofmútbig einem $2 e$ fuch bie sant, won weldem fie fid bie grofs= te Şoffnung verfprach; : benn bie Quinteffens

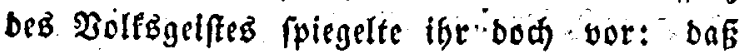
baz - Bolf burd biefen 2erfuch regentrit, baz Mifter für gang Deutfălano abgeben bủrfte!

Alfein es ging und geft unfern Geiftes. ariftofraten, wic bem gutten S S aibo m. Sachs beit blefer berubinte 2ntiqua verfichete: baß er bie berloren gegangene Sonfunf ber Alten wieber bergeftedt, erbielt er einen Ruf an ben

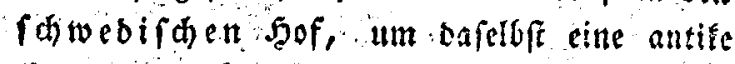

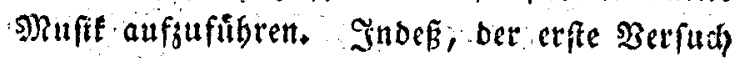
belebrte alle Zübórer, bä zu biefer alten Miu= fit auch alte Djren gigóren.

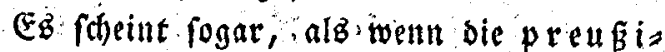

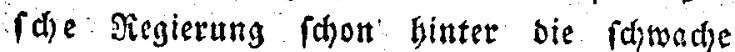
Seite Der beut fof)en (Seifesantiftofratie ses fommen ift; bies berrathen bie mandherlet $\mathfrak{B}$ - orbangen, weldje son ifr feit fur gem cmanirtent - Ea wăre nue fa winfden, bab fie ganj von ber : $2 B a b r b e i t$ fich libergengte: Dá berjenige politifde Beift, bem bie Esiftebariftoknten

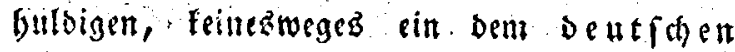
Dolfe argemeffenet fei.

Man erzăblt von bem verwiefenen Siontig

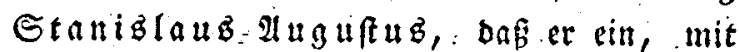
bơchferigenen Şănden ertapptes Berpenft, wets

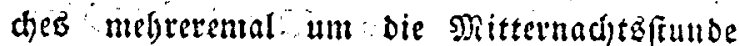
mit feinert. Fivd deinungen ifn-verfolgte, aus beut : Fenfer mit bent Borten binausfturgte: "Ebe id bir glanbe, boffebft du mir bie pros be. Sift but ein wabrer Eecift, fo frigft du gen Şimmel; bift du ein falicher Dămon, fo "magf bu bcim Strube wievergegeben peill."

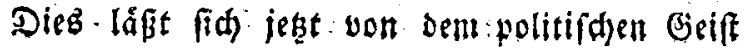




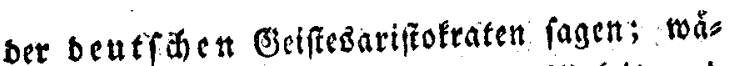
re ber politifde Geiff; beffen Seiligfeit unb

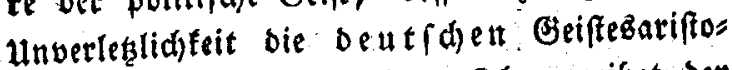
fraten mit fold)er Şingebung fid getweiffet, ber wahre in Deutfdylando Dole Gerrfdente politifde Beift; fo wirbe Steffeng nid)t ungeftraft feinen politlfden Siatobámon baben emaniren laffen bưrfen, bet wie ein Mephis froples gecignet ou fein fdeint, buta Bwietracht bem Buns ber beutfden Geiffes: arifostratie Untergang $\mathfrak{b}$ bereitent.

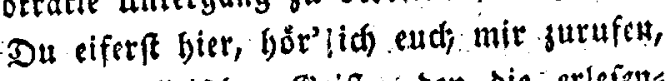
gegent ben politifden Geif, ben die erlefan=

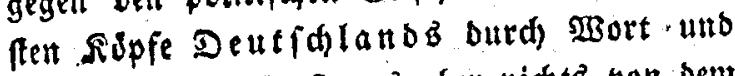
That ergårten, lagft unis aber nidhts yon bem politifá)en Geift, ber Deutfdhlande Dolf bejeelen mag. Doer glaubft bu etwa gar, báa

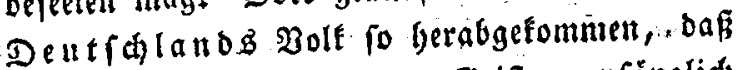

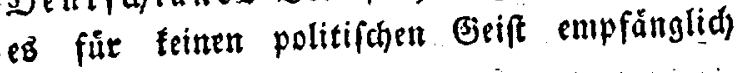
fei?

Rad Dem Jang, weldier Deutfdland bier in feiner \$̇intfamfeit für bie Entwickelung einer Weltbuirgerlidfeit ober Mentahgeit ange: wiefen worben, buirfte fid aud ergeben, wels d)er politifde. (bieift aus feinem Sdjoobe fid fu entrwickeln vermag.

Eprade und gemeinfamer boben find bie feiben $\mathfrak{b e b i n g u n g e n , ~}$ weldje bie: Deutiden!

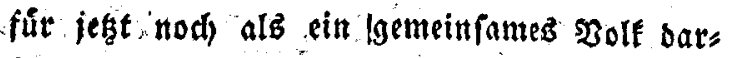
ftellen. bei weitem find bieß aber nid)t bee - Singungen, welde alle Jntereffert einfaliesen, bie in ein Bolf eingreifer. Berfaffung, Sits ten und Religion, find ebenfallz Attribute eis

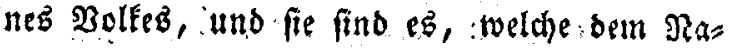
tionalgeift bes Deutfden vorguglid) eitre verfdhiebenartige Ridtung gegeben, indem fie itt ben weit ausigebehnten befanen Deat $(\mathrm{c})=$ Ia ll b s eine maunibfadbe Geftaltung angenom: ment. Sie find es, weldye Entzweing in bem Geift béz bellthen solfez ver: pflangt, und feine Siraft, weldhe bie Eingeit Inur gu erfalten verinag, fo beruntergebradjt, bấ fie allmăblig an innercr Energie vertor, uno

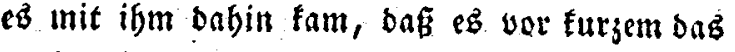
Dpfer einer 3wingherrichaft wurbe.

Diefeż Sdjickfal uon ber glation für bie Folge absuwenben, ift baż Streben jebes ed)ten Patrid: ten. Intee wirft bei biefem Gtreben nid)t bie Pationalität allein, fonbern aud Intereffe mandjer $\mathfrak{A} \mathfrak{r t}$.

Die, ůber Deutfhland ausgebrodene

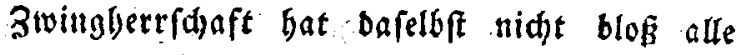
:Rationalitặt mit bem untergange bebrobt, fons Dern aud) bic Grundefte dez Etaatzinobl= ftandes erfdalttert. Der letzte umftand ift es vorjuglid, Der bie Swingherr(d)aft nidjt alfeit 


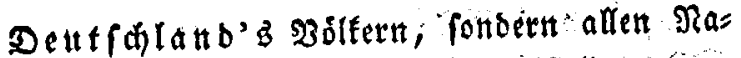
tionen ale láftig unb orưdéno auffellte.

Eg bleibt Daber inmer eine fdrwietige Sufgabe, für Deutfdland eitt Nittel aufs sufittben, woburd feine : Nationalfraft fo ton= zentritt arbalten werbe, um fein gemeinfamed Snterefle gegitt jeden feiner segnel aufredit zat erbaltert.

Die nod jef̧t veftehenbert Berbåltuife,

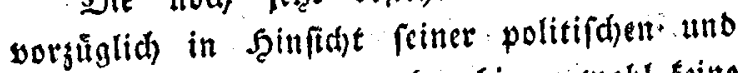

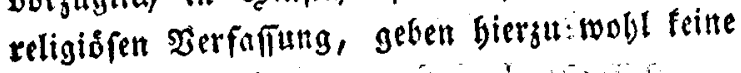

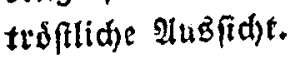

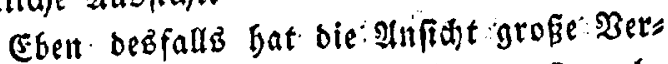
efrer, bã bie Sultur ber beut fdyen Epradhe

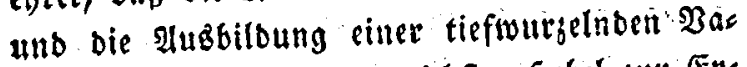
terlangstiebe, bie foigenreidffen f̧ebel jur (Ex

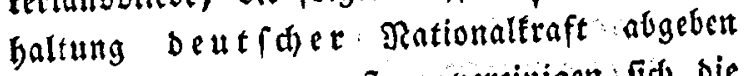
birfter. Zૈlt bem erfern vereinigen fid) bie beut (d)en Purifen; jum Ietgeten bieten bie beut $t$ áen Seftaurationsprebiger. igre. Dents fraft-ahf.

Inbef́, beiber Sefteben. Iiegen Prinzipien

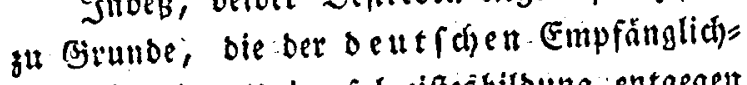
feit für eine univer ralgeifesesbilbung entgegett

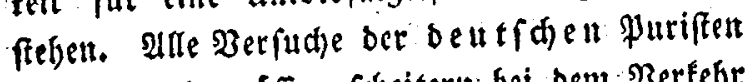
werben und míffen, fafeitertr bei bem : Berfebre

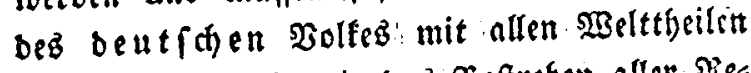

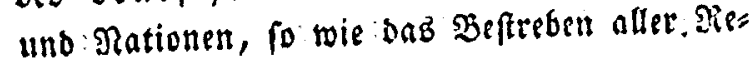

fautationêprebiger, gegen ben in beut $/$ d)er Gemútblidłfeit fid) emporgebrằngten weltbút: gerlidyen Sinn.

- Es würbe bie Grensen, bie wir ber Ent: wickelung !unfers Saifonnementż bier geftellt, $\mathfrak{u}$ berfdreiten, wenn wir bie bier gegebene $\mathfrak{A n}=$

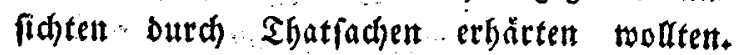

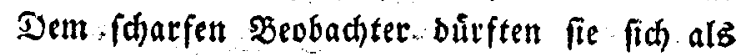
Selege. biergu auforingen, unb biefe múffent

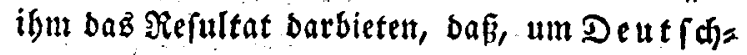

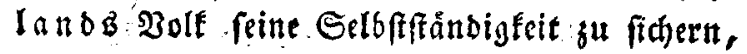
ith ilgm ein : politifder bieift gepflegt werbent muE, Der feiner Beiffésbildong zufprid)t. Der

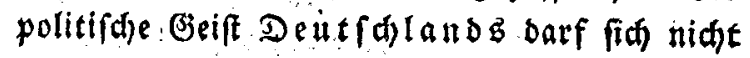
alif fold)e Ericheinungen bafiren, wie bei ans Dern $\mathfrak{B o ̛ l f e r n , ~ ( E r ~ f o l l ~ n i d ) t ~ u r f p r u ̈ n g l i d ~ a u s ~}$ pofitiven Mitteln, wie Spradse, Religion and bergebrad)te. Sitten fid entwickeln; fondern ausi, bcr Quelle, worauz fich aller bieifit genes rivt, aub Der. Jobe, Die Wourjel ber Allgemein= ghiltigkeit ober der untwandelbaren Gerę̧gebung Der Bernunft. Es foll ein.wabrer politifher Bieift frint: : ....

- Fár biefe Infidt fpridft ber Gating Der beutfiden. Geifteabbildung. Fben ourd bie vorginglid)e. Theiluagme ber beut $\{$ d) en. Nation an ber univerfell fortgefdrittenen \$iloung ber Menfdabit, gat fie oas Intereffe für alte sie 
62

Eigènbeiten verloren, welde bie Natur ifge alo Sationaleigenfeiten verlieben. Dezfallż iff fie unter aflen Rationeu biejenige, an weld)er ber

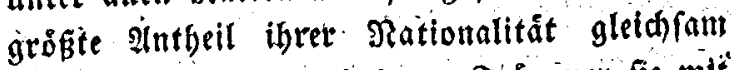
verwifht worben, uno beren Srummer fie mit ciner gewiflen Refignation nod bingiebt.

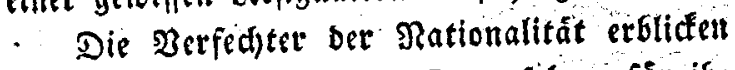
aber in Diefer Ilpathie ber Deutfiden fü its: re Rationalitát ben Urfprung aller ber, in ben leţten Degennict äber fie ergangenen Salaut= tăten. Allein fie lebent in simem tiefen Srr= thirm. Gie verkennen gang ben Geift, ber bie beut ithe Sultur belebt, unb indem fie babin tendiren; bie Deutfden Durds poftibe mit: tel für Srationalitåt wieber empfänglid su mas d)en, arbeiten fie eben ben Abfid)ten Det Natur entgegen.

Inbeß barf es̆ nicht befremben, toenn vors füglich proteftantifale Siegierungen ben 'Planen'

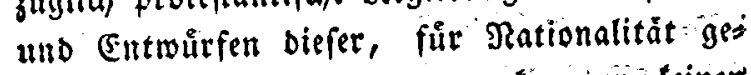
ftimnten Politifer, entgegen fu fommen, teinent sinftand findell. Sie find des Glaubenz, Dá eben bie in oen. Nationen fortgefarittene-unts berfalfe Giciftesbifbung basjentige Siazima ver breitet, woourdj bie fra bei ben $D e u t f \mathfrak{d}$ en eine Empfănglia)leit ge

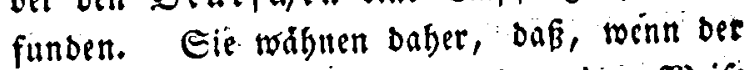
Deutfde wipoeruin zu feiner vorigen झeife
63

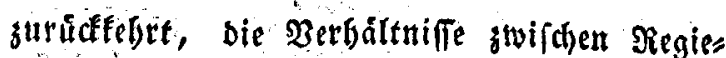
rungen uns regierten endid) ing vorige Ges Ieffe-treten-wurben. - Sic erblicfen baber in ben Pationalitåtş ffauratoren ibrer Mad)t und iftes Einfluffez, und fuchent nun im Einverftandnis mit ibnen bie Deutfden ju reftauriren.

Den vorgưglidjen Grundftein bierju, glaus ben fie in einen Alufwanb von Mitteln zur. ferftellung ber Pietait ober diriftichen Fróm: migfeit im Gebiet bes ProtefantiBmus fu les get. Amein biefes sittel beiliget feinesweges ifren 3wed. Mogen fid bod biefe religidfen Enthufinften fuetf beutlid) beftimmen, was fie unter d)riplid)er Frómmigfeit verfteben, uno endlid) genna aus ben Benebmen unb ber

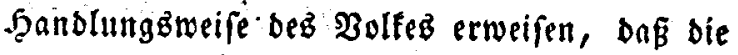

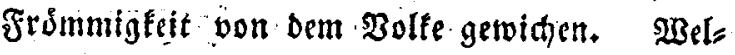
đ) be ut f he Bolf in bem Raufe bet orüctenden Erniebriging Deutfalanos zeifen? Hub ftelle mañ bod eine: Bergleid)ung jtvifd,en ber, Iat ber Serbred)en und ber Zabl ber Berbres d)er frubberer Beiten unb ber ber neneren Beit

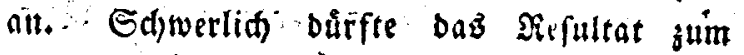
গRad)tbril ber leftern aubfallen.

Die Regierungent leben gewís in einem tiefent Jrutbum, renn fie bes Gilaubens fiat, 


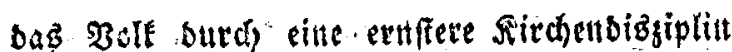
in feiner Denfart gu begrănjelt, Die. religios

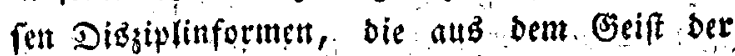
Sieligionblebret-bervorgeben, Gaben nod nie Die Dentart bez Bolfé geleitet. Sie múffen cin. Sejultat ber. Solfabenfart fein; . Dant be= wirten fie simen Stilltand und ein bebarrlidjes faften an cintr Form. Shlögt man : einen

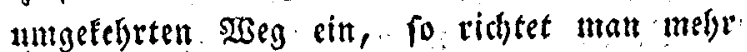
Gajaben an, alz man Gutez, th Gewirfen ver mcint.

Mant erimnere fid bod nut an bie Mir: fung, die vormals bas preufifde. Rel $i=$

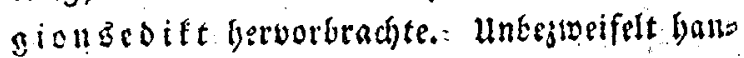

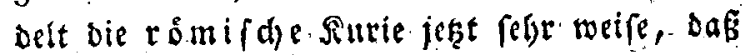
fie bem Dolfe in Sinfid)t Der religiofen Sultur feine ftrenge Disjiplin verleibet, ob fie gewis

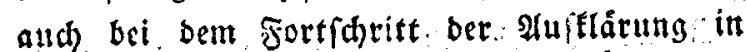
fatholifd)en. Panden biergat megr Beranlaffung,

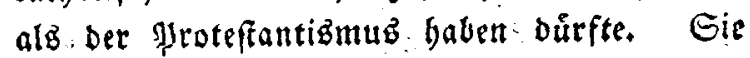
fennt ben Geift ber Zeit, uno, iff úberjeugt, oaß ar fíd nid)t in Gdranten jurůdtweifen lågt, betten et entwadjen.

Die falte Defonnenbeit ber feitigen $r \$ m i s$ (d) en. Purie fellt bem Seobad)ter einen nitht unintereffanten. Suntraft, gegen bab jestige pies tifrifde Sienegmen ber proteffantifden Regieruns gen anf. An Eeften uno Partigen war ber

Fre?
Broteftantiỏmuz vermóge reitter Senbeng int: met reid). NGer hie batte $P B$ in feinem Sdook

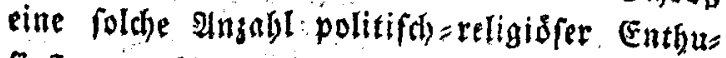
faften genabut, als man jebt auf f̧och (ct)ulen und Seanjeln und in mand)en Sdyriften fid ausfprechen bort, uno bicfe glauben fid in ibrem Benefunen beftånft, ourd) Den pietiftifich Inftrid), ben bie Regierunget bafelbfin in sen

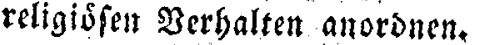

Diefe Partbei will nun ben politifdert Selif ber Deutfden mit ben pofitiven Blan: benzanfichten, beB \$rotefantisimus in Berbin= oung feren, unb fudt butd diefes band gleid =

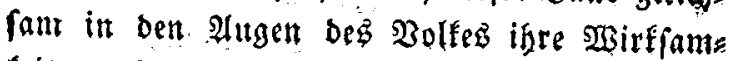
feit zu beiligen. Sie fieft im protefrantibs mub die Duelle für die Deduttion aller fante: wiffenfdaftlidben Soeen, welá)e bie franjệfifhe

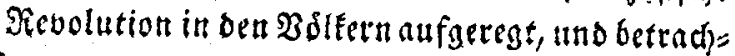
itet igu als oen fidjerfen ftellnng und Erfaltung Derfelben. Sif will alfo ben Proteftantismuz als die cingige Ouelle bss Seils, fur Deutfdiand anffellen, unb nur bem, sas ben Anfichten und Crunezugen vef feloen entfpridft, foll als. Deut fáer politifíper Geift gebuldigt werben.

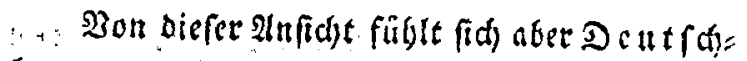
Iand Bolf nidht üborseugt, uno indem pitt faer won proteffamtifarn Entbufiofen burd 


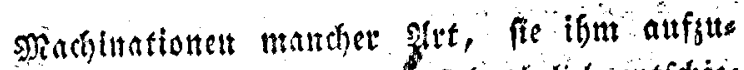
sringen fud t, erbătet es fid tăglids entidjes beter: dás ser proteftantifde "Boben eigentlids eine Suefle univerfeller Geiftesbilding bet গgenid)brit iff.

Sebr fdrandych Einfluf baben Die Madjis nationen bir Sationalitätseffauratoren. Das Bole belăt fefr ben Etanopurft im Sluge, auf weldett eb bie Drangfale bet Beit bingebrangt uno voul biefent nus tif ifim bas siefultat ges worben: Daf bes 20 lfes seil 'mer gefidert werben Eant, nidt went ber Denfart, fons:

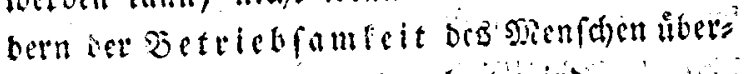
bawpt sin feftece Girund gelest niro.

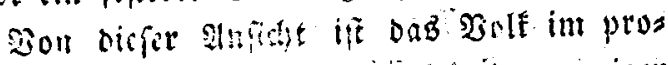

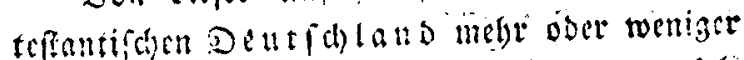
befelt, ans fúblt bab Bobưrinf, fie verfols gen ju fountelt, nadjoridelidher, als irgeno sin anorres. Lino warmm? Sacil bie Betriebfom

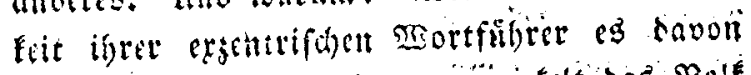
abjubringen fudut. Sman uimgnutelt bab BolE

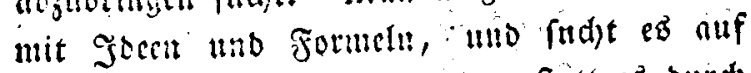
ideale Regionen sll verffeligen, ftatt es burd) ernfre und cingreifende Jufitutionen ju frifs tigett.

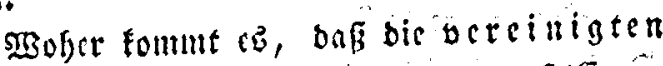
Staaten in s!merifa gebeiben? Dab Engs lano ful folther goben Bolfomacht fid empors geboben? : baf Franteich fhout sine gludfliche

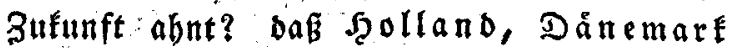
unb Sdroben ber Fruchte ber Geiftezum: toâlyuth in Europa theilhaftig werben? -

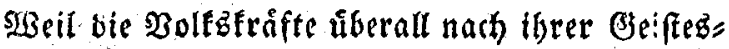

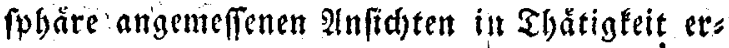
balten werben. Aber in $D$ entifd land?

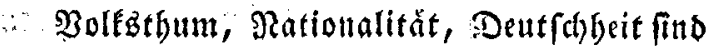
bie Feţen, um weld)e fich tïglid pin netter Seaufen Rampfluftiger fammelt, und von bes rell Gladiatorentinften Segierung uno $\mathfrak{B}$ olE irre gt werben, tăglid) in Gefaly feben.

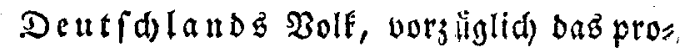
teftantifale, ift nad meitrer Arificht $D_{a b}$ vers

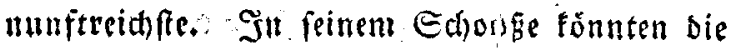
ftaatzimiffenfdaftliden Josen, unt welden ber menifdlidbe Geift - burd bie tollbare politifd)e umwăljung bercidjert worben, in ifrer fdưn: ffen Sblutbe und im vollfemmenften (jedsiben aufgeben., Illein man fucht biltch einen. Galli= matbias oon pofitivem Soentram biefe fabo: nen Alnlagen fu Depraviren.

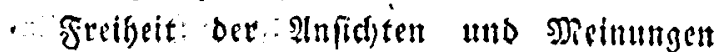
fiber ocu allanmengang Des frligeat uno zeit: lla)en Rebens, bie fidh fo mannigiad) in ben verfhiedenen Religionen auŝpricht, ift gewis

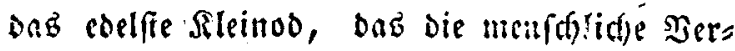
nanft im faufe ber politifáden umwăijung แแ 
68

ferer 3eit fu wirkbigen gelernt. Poot allent Siationen witb ifm wentgftens fillfd)weigend gefulbigt. 2lber in proteftantif(den Deutfas $\mathfrak{l}_{\mathfrak{a}} \mathrm{b}$ if es, wo man burd bialleftif(d)e Ulebunts gen bas : Bolf bagegent abjuftumpfen fud)t.

ßutr Durdh. Die Siltur jener Freibeit forna neit Sonftitutionen Gerborgehen, in reld $)$ en er: folgenreich bie 马oeent wurjeln fönnen, bie bent

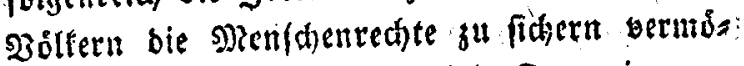
getl, um welche fie fo viele Dezennien ges blutet.

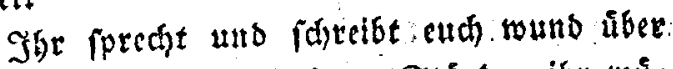
bie Exiffenz der privileginten Stánbe, iljr twis= tyst gegen bie Ariftofratic ber Befurt. Sft

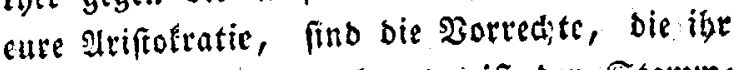
fir eatre slnfictiten verlangt, if der Etamm= baum, Den the firr ifn worgeigt, anbern ltea fpeungas? SBird er baltbarere Orunde aufferts Isn? Sff er vid)t viclmegr bce Urquell allev Ilngleid)beit bes sred)tb, nid)t allein für bie

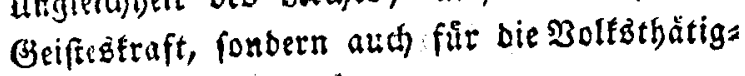
teit?

Deuthalanoz şolf ift auf eine Etufe ber Sultur fortgefdritten, um bie Spradje ber gefeęgebenden פernanft ju faflen. Sied)tlidje Grfinnungen und ausbanernbe Betriebfamteit bat es in ben Zeitent ber Drangiale unter dens Jodie Iev 3rwinghecriajaft erbärtet. . Diefe
69

burd) gute Gepetze und Inorbnungen zu forits= sen unb gu erfalten, ift bas einjige Mittel,

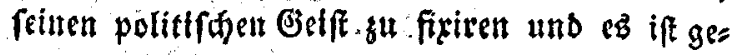

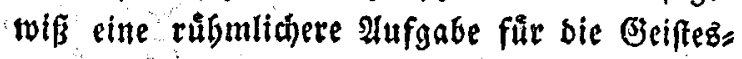
ariftofraten DeutfalandB, biefe gut rafaffen

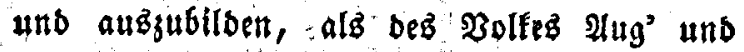
Dbr mit Mbantażmagorien and veralteten For:

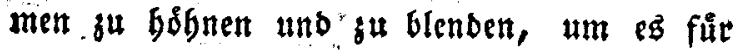
einen politifchen Geift empfänglid z̆ madjen, Der ez von feinem grofent, ifm von ber Ratur angerwiefenten Bernf abbringt: Die $\mathfrak{B} \&$ lfer za lebren, Dá bie Entwidelang bet gefeggebenden Bernunf bie Quelle

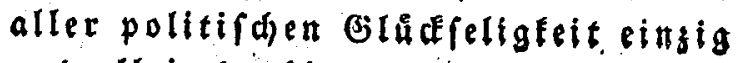
und alletin baebletet. 